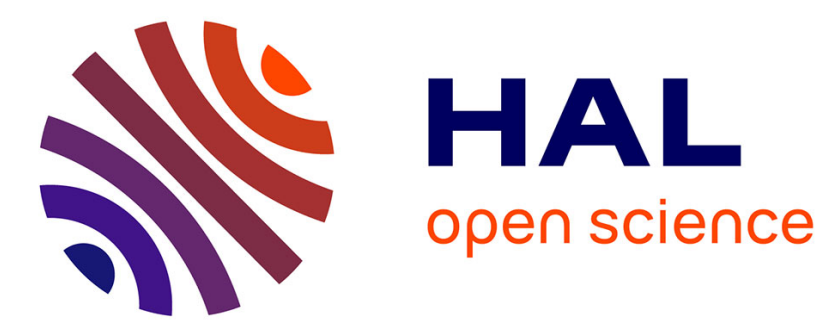

\title{
Protection du niobium contre l'oxydation
}

\author{
P. Stecher, B. Lux, R. Funk
}

\section{To cite this version:}

P. Stecher, B. Lux, R. Funk. Protection du niobium contre l'oxydation. Revue de Physique Appliquée, 1970, 5 (3), pp.573-595. 10.1051/rphysap:0197000503057300 . jpa-00243430

\section{HAL Id: jpa-00243430 https://hal.science/jpa-00243430}

Submitted on 1 Jan 1970

HAL is a multi-disciplinary open access archive for the deposit and dissemination of scientific research documents, whether they are published or not. The documents may come from teaching and research institutions in France or abroad, or from public or private research centers.
L'archive ouverte pluridisciplinaire HAL, est destinée au dépôt et à la diffusion de documents scientifiques de niveau recherche, publiés ou non, émanant des établissements d'enseignement et de recherche français ou étrangers, des laboratoires publics ou privés. 


\title{
PROTECTION DU NIOBIUM CONTRE L'OXYDATION
}

\author{
P. STECHER, B. LUX, R. FUNK \\ Institut Battelle, Genève, Suisse
}

\begin{abstract}
Résumé. - A dessein de protéger les alliages de niobium contre l'oxydation à haute température, différents types de revêtements à base de siliciure ont été élaborés.

Dans ce rapport, il est discuté de questions générales concernant les propriétés requises et, en particulier, des problèmes que posent la fragilité des couches protectrices et l'apparition de fissures.

Une description y est faite de la structure et de l'endurance de différents revêtements qui assurent une protection au niveau des fissures par l'association de la couche de siliciure poreux à une phase mobile autoréparatrice incorporée dans les pores.
\end{abstract}

Il s'agit des systèmes suivants :

- siliciure $\mathrm{Nb}(\mathrm{Ti}) \mathrm{Si}_{2}$ - alliage métallique $\mathrm{Sn}-\mathrm{Al}$

- siliciure $\mathrm{MoSi}_{2}$ - verre de borosilicate

- siliciure $\mathrm{Cr}, \mathrm{Fe}, \mathrm{Nb}$ - oxyde plastique formé par le siliciure lui-même pendant l'oxyda-

- siliciure $\mathrm{Cr}, \mathrm{Ti}, \mathrm{Nb}$ - alliage métallique $\mathrm{Sn}-\mathrm{Al}$.

Ce dernier système de protection est décrit en détail ; il se distingue :

- par une fabrication selon une méthode (c slurry ) qui permet la réalisation du revêtement sur des alliages de niobium de différentes compositions, dimensions et formes,

- par une faculté de guérison, à températures élevées, des fissures dans la couche de siliciure grâce à l'action de $\mathrm{Sn}-\mathrm{Al}$ liquide.

Dans ce texte sont décrits également :

- le procédé de fabrication de la couche de siliciure par projection d'une poudre de siliciure de chrome-titane et fusion du dépôt à températures élevées,

- l'imprégnation par traitement dans un bain de Sn-Al,

- les résultats obtenus lors de différents tests d'oxydation, y compris des tests d'impact et de choc thermique. Il s'agit particulièrement de l'influence de la rapidité des changements de température sur l'endurance du revêtement dans les tests cycliques.

- les modifications du revêtement par oxydation et diffusion, qui affectent le pouvoir de protection du revêtement.

Abstract. - In order to protect niobium alloys against high temperature oxidation, different types of coating based on silicides have been developed.

The general questions of the required properties are discussed, in particular the problems of the fragility and fissuring of the protective films.

The structure and endurance of self-healing films are described. In these films protection in the event of fissure formation is obtained by incorporating a mobile phase in the pores of the silicide film.

These systems are :

- $\mathrm{Nb}(\mathrm{Ti}) \mathrm{Si}_{2}$-silicide

- $\mathrm{MoSi}_{2}$-silicide

Sn-Al alloy

- $\mathrm{Cr}, \mathrm{Fe}, \mathrm{Nb}$-silicide borosilicate glass

- Cr, Ti, Nb-silicide Sn-Al alloy.

The last system is described in detail. It has the advantages that :

- the layer can be formed by a slurry method allowing niobium alloys of different compositions, dimensions and shapes to be coated,

- the healing of fissures at high temperatures is good, owing to the action of liquid Sn-Al in the silicide film.

There are also descriptions of :

- the fabrication process of forming a layer of chromium-titanium silicide by powder spraying, followed by a high temperature fusion of the deposit,

- the impregnation process by treatment in a bath of $\mathrm{Sn}-\mathrm{Al}$,

- the results of different oxidation tests including tests after impact and thermal shock resistance. In particular the coating endurance in thermal cycling tests has been studied as a function of the rate of change of temperature,

- the modifications of the coating due to oxidation and diffusion which affect its protection capabilities. 
Introduction. - 1. ProblèMe GÉNÉRAL. - Les alliages de niobium sont doués d'excellentes propriétés mécaniques à des températures où les superalliages ne sont plus utilisables. Par contre, ils présentent l'inconvénient que leur utilisation en atmosphère oxydante requiert un système de protection superficielle.

Or, le choix des matériaux qui peuvent être retenus pour des couches protectrices est restreint. Les revêtements d'oxydes posent des problèmes d'adhérence, alors que les revêtements métalliques diffusent trop rapidement. Ces deux types de revêtements n'offrent qu'une protection de courte durée.

Seuls les revêtements à base de composés intermétalliques qui se couvrent de films protecteurs d'oxydes se sont révélés réellement aptes à répondre aux applications requérant une protection durable à des températures allant jusqu'à $1400^{\circ} \mathrm{C}$.

Actuellement, les meilleurs revêtements pour des alliages de niobium sont à base d'aluminiures ou de siliciures de niobium. Les siliciures, qui sont doués d'une plus grande stabilité thermodynamique, sont supérieurs aux aluminiures en ce qui concerne la durée de protection (voir Fig. 1, [1]). Ces composés confèrent aux revêtements, outre une résistance élevée à l'oxydation, une bonne adhérence au substrat et ils diffusent assez lentement dans le substrat.

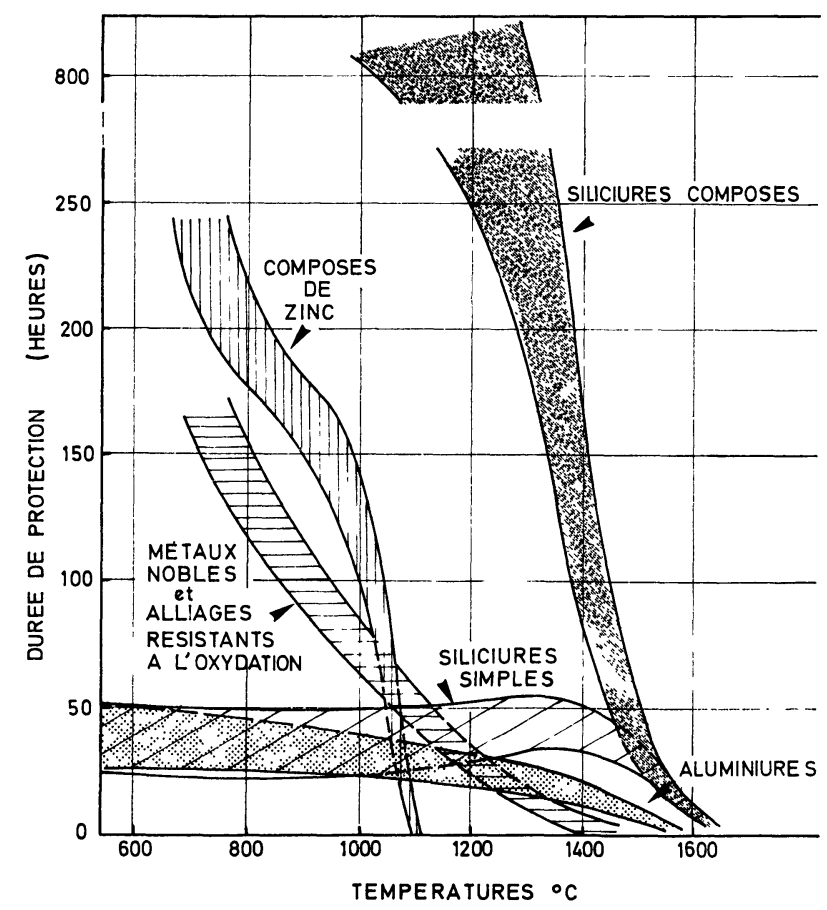

Fig. 1. - Durée de protection de revêtements de différentes natures sur des alliages de niobium, en oxydation cyclique, d'après J. Gadd [1]

Par contre, le manque de ductilité de ces composés intermétalliques qui est responsable de la fissuration du revêtement lors de changements de température, constitue leur défaut majeur. Les fissures ainsi formées représentent les points faibles de ces revêtements et provoquent des défaillances locales avant l'épuisement complet de la couche par oxydation et par diffusion dans le métal de base. La mise au point de revêtements auto-réparateurs permettrait de prévenir cette défaillance prématurée et de maintenir le pouvoir de protection en dépit de fréquents changements de température.

Nos travaux sont orientés vers le développement de revêtements où la protection au niveau des fissures est assurée grâce à l'incorporation d'une phase mobile auto-réparatrice dans le composé intermétallique $[2,3]$. Ces travaux ont abouti à la réalisation d'un revêtement de siliciure imprégné d'un alliage d'étain-aluminium.

2. ApPlications du ReVÊTEMENT. EXIGENCES ReQUISES. - L'utilisation d'alliages de niobium à haute température pour des réacteurs d'avions, des moteurs de fusées et des engins hypersoniques exige un revêtement de forte endurance. Il existe d'autres cas d'applications industrielles où une endurance extrême ne joue pas de rôle primordial (tableau I).

Parmi les sollicitations auxquelles est soumis le revêtement et qui dépendent du genre d'applications, on peut relever :

- l'oxydation et la corrosion prolongées à haute température,

- les changements de température répétés, rapides ou lents,

- la dilatation et le fluage du métal de base,

- l'érosion due à des gaz chauds circulant à grande vitesse,

- les impacts mécaniques de particules solides,

- les forces centrifuges,

- les basses pressions d'atmosphères.

En raison de leur fragilité, les couches simples de siliciure sont sensibles aux variations de température, aux tensions communiquées par le métal de base et aux impacts mécaniques, et elles font apparaître des fissures et des défauts. L'introduction d'une phase mobile permettrait de neutraliser l'effet de ces défauts et de compenser ainsi l'absence de ductilité.

Par contre, le problème de la rétention de l'élément auto-réparateur reste entier dans le cas de l'érosion par des gaz à grande vitesse, de la présence des forces centrifuges ou même celui de faibles pressions.

Nous avons tenu compte de cet aspect du problème [4] en logeant la phase mobile dans une couche intermétallique poreuse.

3. TyPes DE COUCHES PROTECTRICES ÉTUdiés. Les types de revêtements suivants, dont le principe repose sur l'association d'un siliciure et d'une phase mobile, ont été examinés :

1) siliciure $\mathrm{Nb}(\mathrm{Ti}) \mathrm{Si}_{2}$ - alliage métallique $\mathrm{Sn}-\mathrm{Al}$,

2) siliciure $\mathrm{MoSi}_{2}$ - verre de borosilicate, 


\section{TABLEAU I}

Applications possibles du niobium ou de ses alliages et sollicitations exercées sur le revêtement

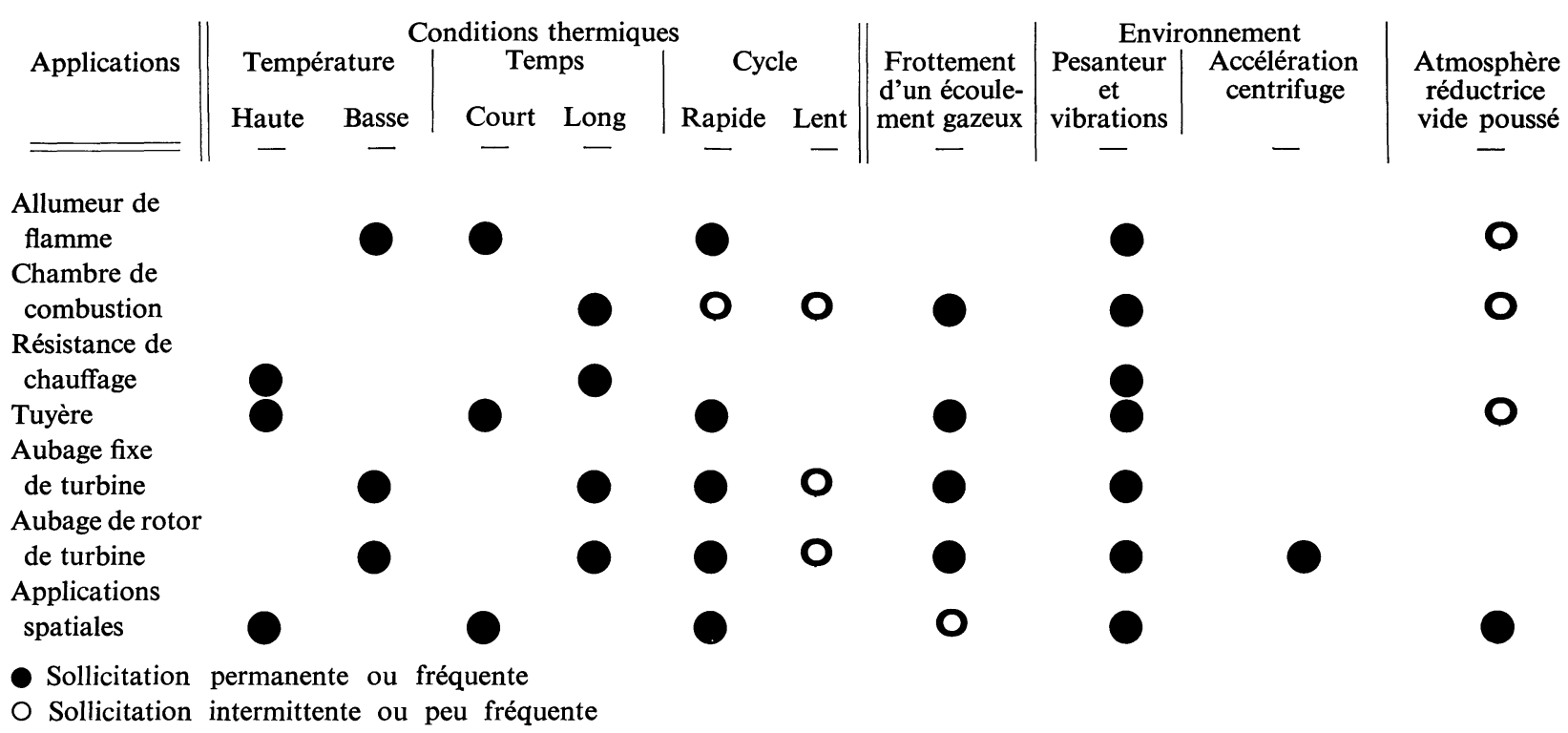

3) siliciure $\mathrm{Cr}, \mathrm{Fe}, \mathrm{Nb}$ - oxyde plastique formé par le siliciure lui-même pendant l'oxydation,

4) siliciure $\mathrm{Cr}, \mathrm{Ti}, \mathrm{Nb}$ - alliage métallique $\mathrm{Sn}-\mathrm{Al}$.

La figure 2 schématise la structure de ces revêtements et indique leurs méthodes de fabrication.

Le tableau II et les figures 3 et 4 permettent une comparaison de la durabilité de ces systèmes de protection soumis à différents tests d'oxydation ${ }^{(1)}$.

Les propriétés spécifiques de ces revêtements se résument comme il suit :

1) Revêtement $\mathrm{Nb}(\mathrm{Ti}) \mathrm{Si}_{2} / \mathrm{Sn}-\mathrm{Al}[2,3]$. — La couche de siliciure poreux, entièrement remplie de $\mathrm{Sn}-\mathrm{Al}$, est déposée sur du niobium pur ou sur un alliage binaire niobium-titane par un traitement dans un bain liquide saturé de silicium.

Malgré la forte tendance à la recristallisation du squelette de siliciure en présence de l'alliage Sn-Al liquide, ce revêtement résiste bien aux tests statiques (test A) $\left(^{2}\right)$ à température constante et aux tests cycliques très rapides (test $F$ ). En revanche, ce revêtement s'est révélé sensible à des séjours prolongés à température élevée alternés avec des refroidissements lents (test C). Ces conditions engendrent l'épuisement rapide de la phase $\mathrm{Sn}-\mathrm{Al}$ par oxydation suivi d'une attaque du squelette de $\mathrm{NbSi}_{2}$ par la peste.

(1) Les différents tests indiqués dans les tableaux portent des lettres majuscules de référence qui sont reprises dans le texte.

(2) Voir tableau II.

Dans une couche déposée sur un alliage niobiumtitane, le siliciure $\mathrm{NbSi}_{2}$ contient du titane, ce qui réduit la sensibilité à la peste et contribue à améliorer la résistance à l'oxydation cyclique.

L'alliage de la couche de $\mathrm{NbSi}_{2}$ par du chrome ou du titane, pendant le traitement d'une base de niobium pur ou d'alliage revêtu d'une couche de niobium pur dans le bain de Sn-Al contenant un de ces deux éléments, n'a pas conduit à la réalisation de revêtements meilleurs que $\mathrm{NbSi}_{2} / \mathrm{Sn}-\mathrm{Al}$.

2) Revêtement $\mathrm{MoSi}_{2} /$ verre. - Dans ce type de revêtement, la phase $\mathrm{Sn}-\mathrm{Al}$ est remplacée par un verre de faible viscosité à température élevée qui, à la différence de l'alliage $\mathrm{Sn}-\mathrm{Al}$, ne se consume pas par oxydation. $\mathrm{NbSi}_{2}$ pur ou allié au chrome-titane, recouvert de verre, s'oxyde à haute température et les oxydes formés modifient défavorablement les propriétés du verre.

Par contre, $\mathrm{MoSi}_{2}$ est stable en présence de verre de borosilicate à haute température dans des conditions oxydantes. Des couches de $\mathrm{MoSi}_{2}$ poreux sont obtenues par frittage d'une poudre de molybdène déposée par la méthode "slurry" $\left({ }^{3}\right)$, suivi d'une siliciuration. L'imprégnation de $\mathrm{MoSi}_{2}$ est réalisée par un traitement dans un bain de verre à température élevée. L'inconvénient majeur de ce revêtement est la faible adhérence du squelette poreux de $\mathrm{MoSi}_{2}$ au substrat, qui est à l'origine de la défaillance rapide du revêtement dans les tests cycliques.

(3) Cette méthode sera décrite en détail lors de la description des couches de siliciure allié au chrome-titane. 


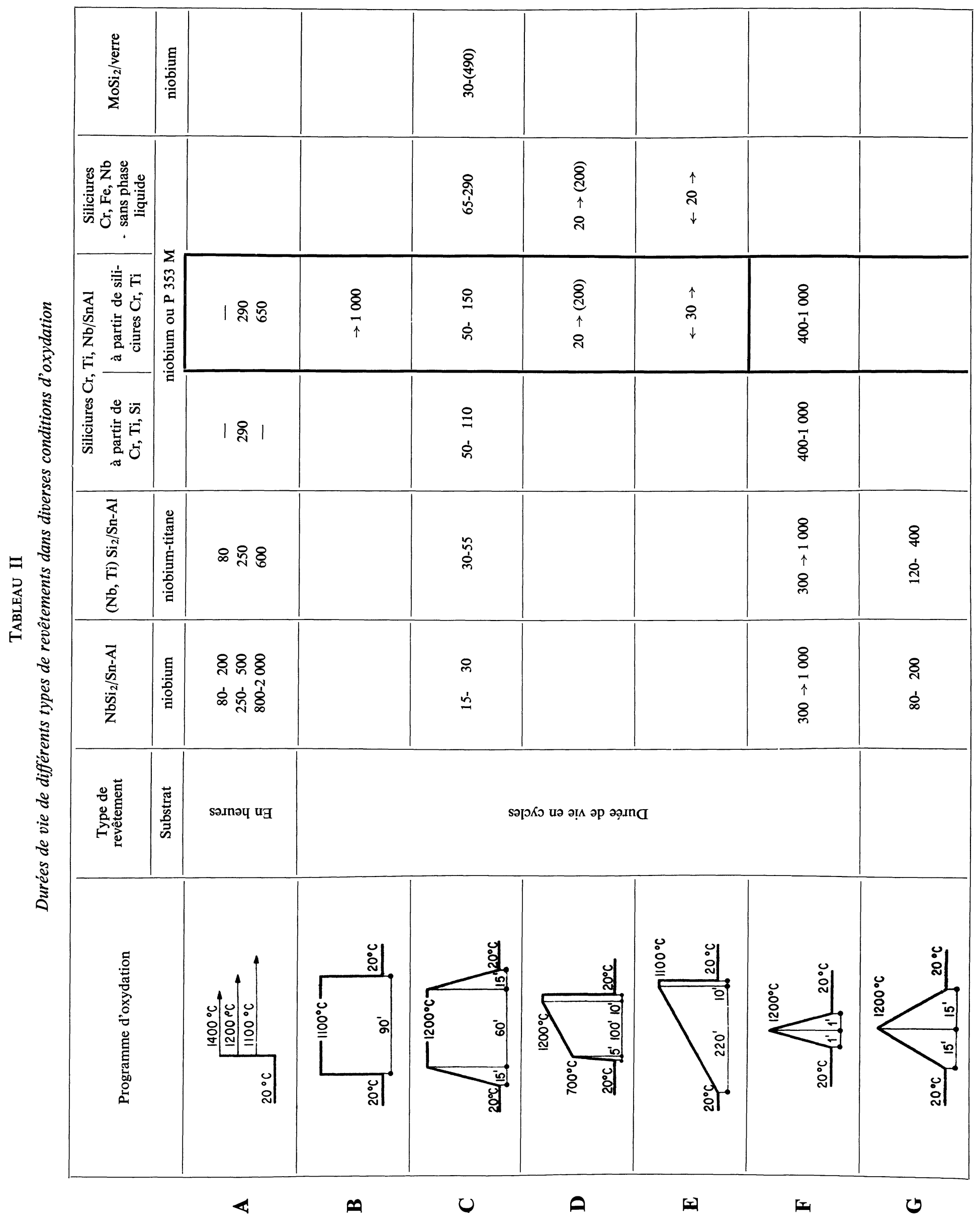




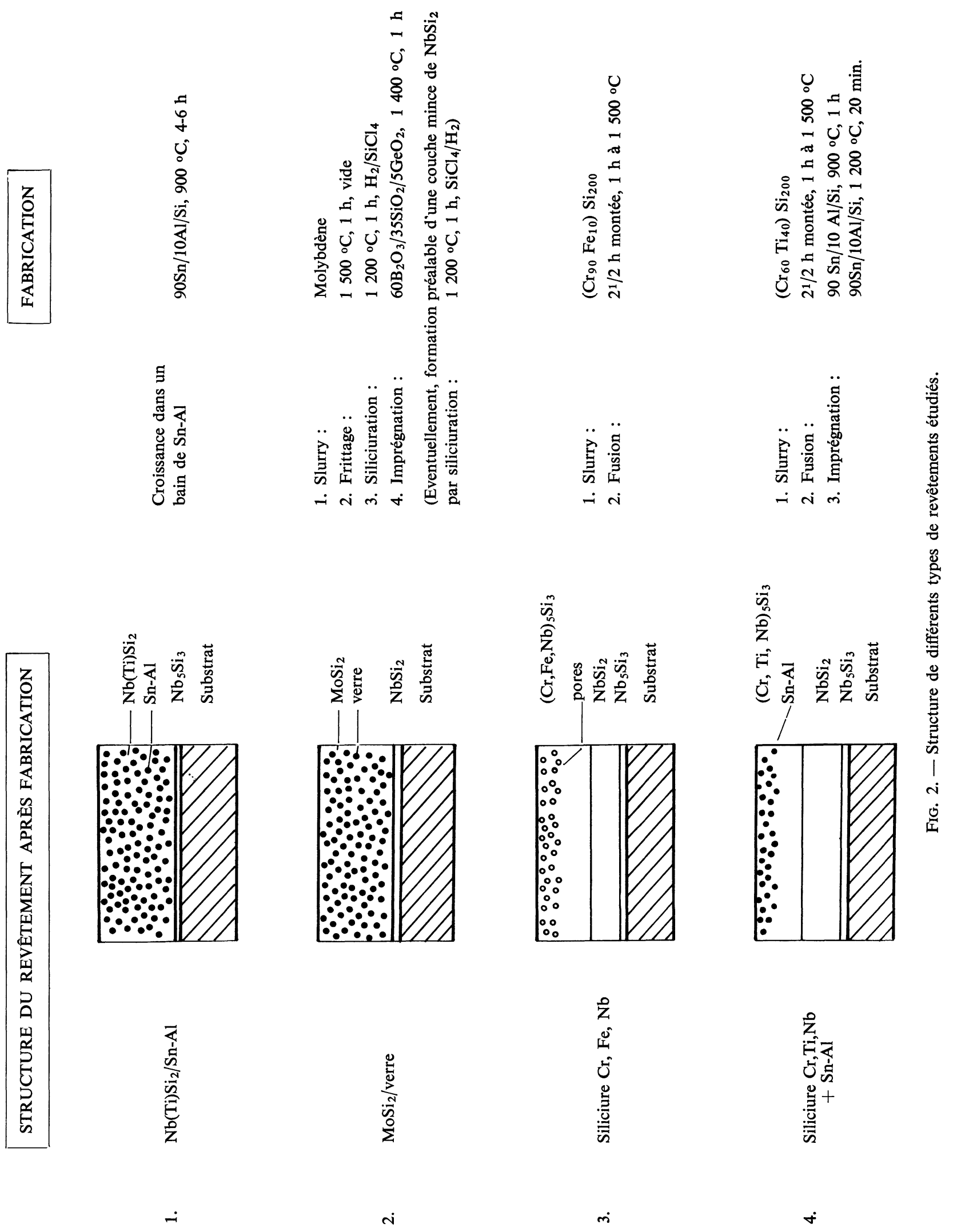




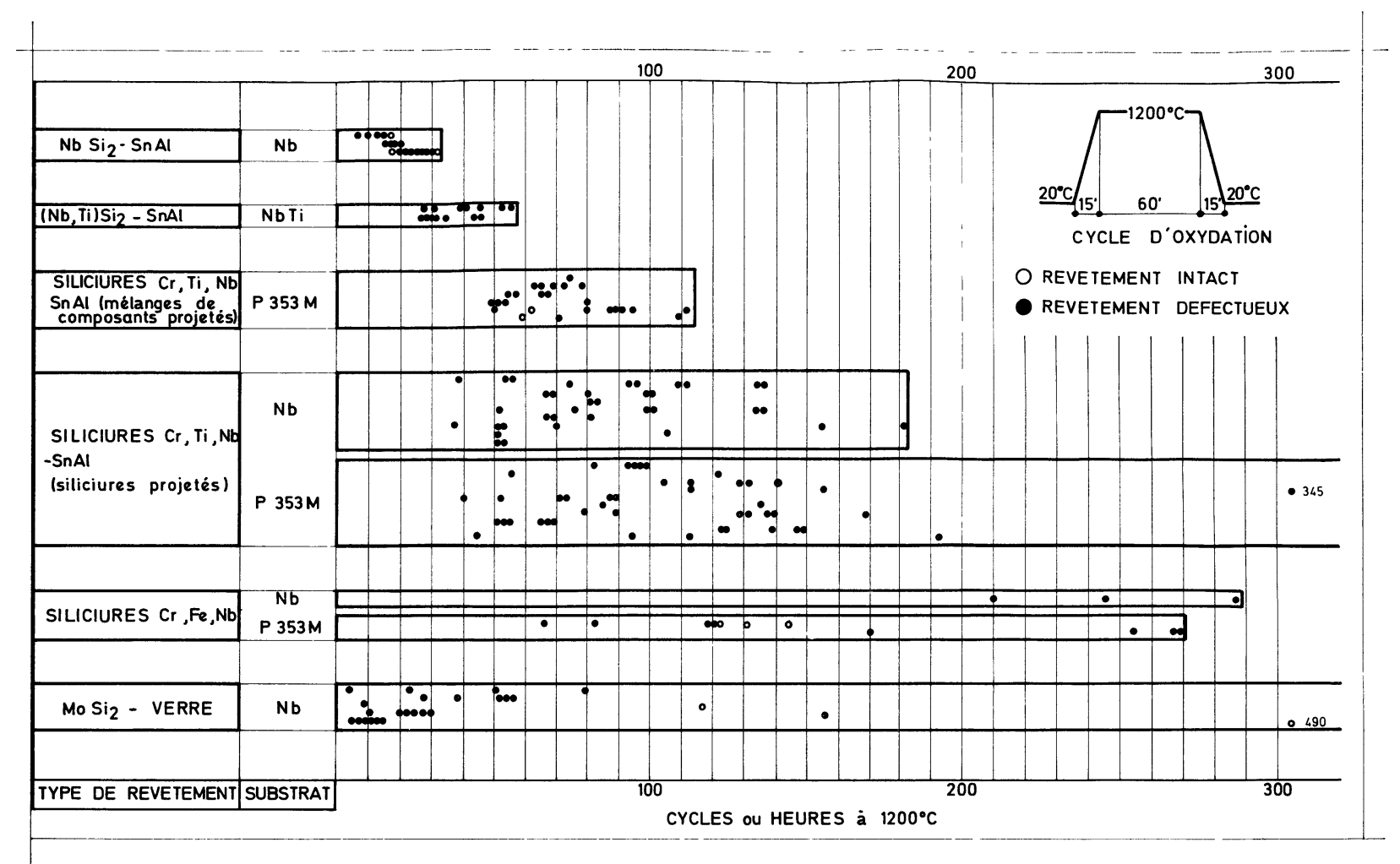

FIG. 3. - Durée de vie de différents types de revêtements dans le test d'oxydation cyclique E.

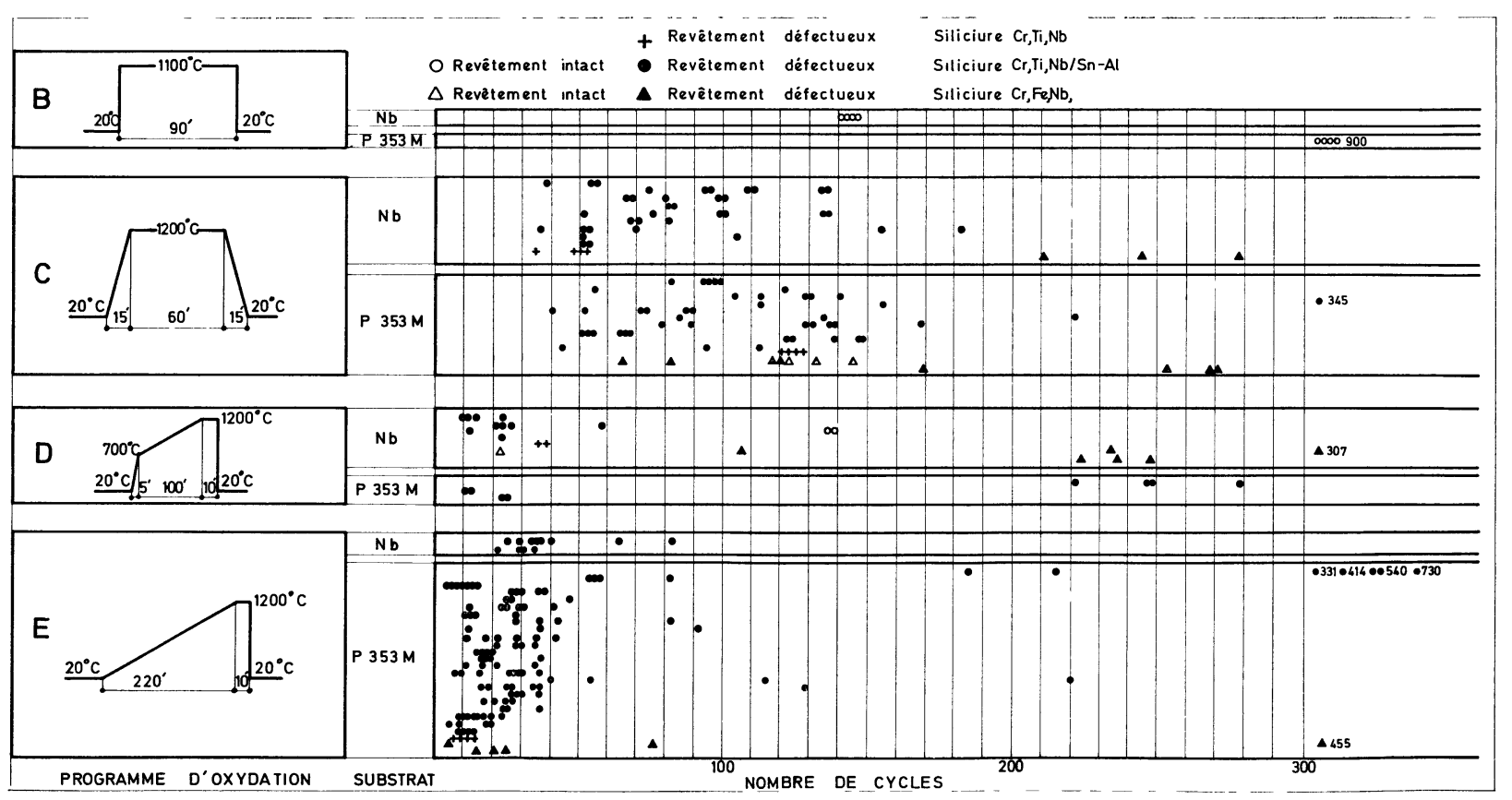

FIG. 4. - Durée de vie du revêtement de siliciure $\mathrm{Cr}, \mathrm{Ti}, \mathrm{Si} / \mathrm{Sn}-\mathrm{Al}$ (siliciure $\mathrm{Cr}, \mathrm{Fe}, \mathrm{Nb}$ ) dans les tests d'oxydation cyclique D, E, F, G.

3) Revêtement de siliciure de $\mathrm{Cr}, \mathrm{Fe}, \mathrm{Nb} .-\mathrm{Le}$ revêtement de siliciure allié de chrome-fer est formé par le procédé «slurry», c'est-à-dire par déposition d'une poudre de siliciure chrome-fer et réaction du dépôt avec le substrat, à température élevée.
La forte résistance de ces couches aux tests cycliques repose sur la formation d'oxydes vraisemblablement plastiques en surface. Ces derniers remplissent également les fissures et empêchent l'infiltration rapide de l'oxygène jusqu'au substrat. 


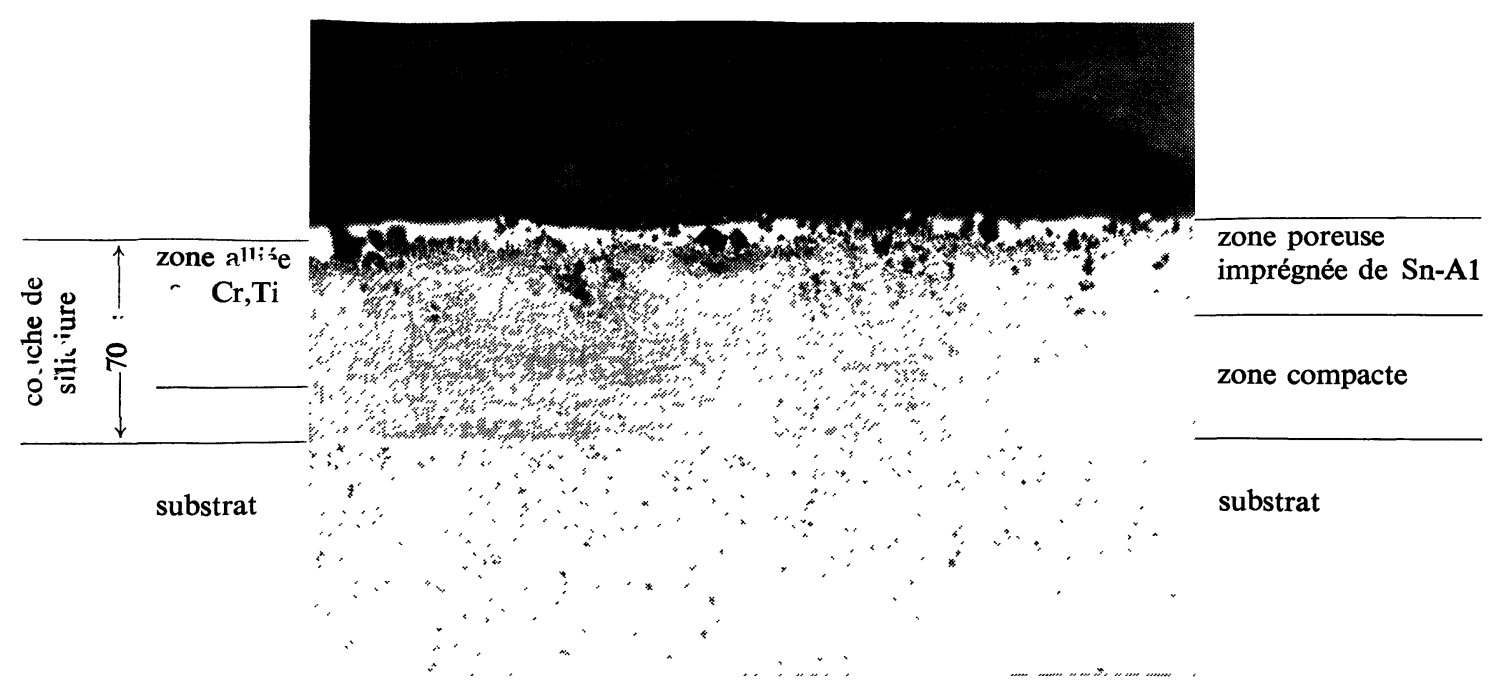

FIG. 5. - Structure du revêtement de siliciure $\mathrm{Cr}, \mathrm{Ti}, \mathrm{Nb} / \mathrm{Sn}-\mathrm{Al}$ après fabrication.

4) Revêtement de siliciure de $\mathrm{Cr}, \mathrm{Ti}, \mathrm{Nb} / \mathrm{Sn}-\mathrm{Al}$. $\mathrm{Ce}$ système de protection est particulièrement intéressant car, à la différence des revêtements mentionnés ci-dessus, une disparition des fissures peut être obtenue dans certaines conditions, dans la couche de siliciure, sous l'action de la phase Sn-Al. Ce phénomène d'auto-réparation assure une grande durabilité dans des conditions d'oxydation qui comprennent de fréquents changements de température. détail.

Ce système de protection est analysé ci-après en

Revêtement de siliciure allié de chrome-titane imprégné d'étain-aluminium. - 1 . STRUCTURE DU REVÊTEMENT. - Le revêtement est constitué de deux composants résistant à l'oxydation :

- une couche de siliciure allié au chrome-titane,

- un alliage étain-aluminium, liquide aux températures d'oxydation.

Le siliciure comporte deux zones: La zone intérieure compacte de siliciure constitue l'enveloppe qui renferme hermétiquement le métal de base. La zone extérieure, qui est poreuse, fait fonction de réservoir de la phase auto-réparatrice et renferme l'alliage de Sn-Al (Fig. 5).

L'association de ces deux composants et la structure particulière du revêtement permettent de réunir dans le même revêtement certaines propriétés des siliciures et des aluminiures.

2. FABrication du REVÊTEMENT. - Le revêtement est réalisé par étapes successives. La figure 6 illustre la technique de fabrication :

1) Déposition d'une poudre de siliciure par projection,

2) Formation de la couche de siliciure par fusion du dépôt à température élevée et sous vide,
3) Imprégnation de la couche de siliciure par traitement dans un bain d'étain-aluminium.

(1) PROJECTION DE POUDRE DE SILICIURE

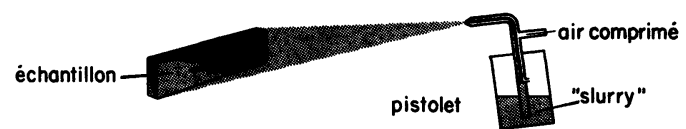

(2) FORMATION DE LA COUCHE DE SILICIURE

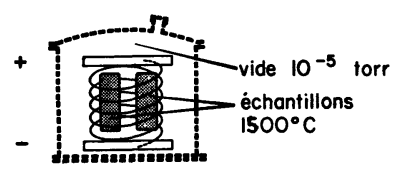

(3) IMPREGNATION AVEC SnAI.

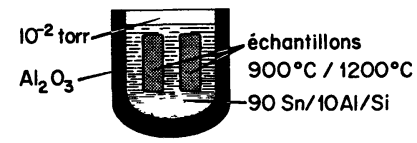

FIG. 6. - Fabrication du revêtement de siliciure $\mathrm{Cr}, \mathrm{TiSi} / \mathrm{Sn}-\mathrm{Al}$.

2.1 Formation de lá couche de siliciure. 2.1.1 Déposition par projection d'une poudre de siliciure. - Méthode "slurry ». - Pour obtenir des couches de siliciure allié, nous avons adopté la méthode "slurry ». Cette technique offre l'avantage de permettre de réaliser des couches à teneur élevée en éléments d'alliage par la formation du revêtement, à partir de poudres contenant les constituants du revêtement final. Les poudres, qui sont dispersées dans une laque nitrocellulosique servant de liant, sont déposées à la surface du substrat par projection au moyen d'un pistolet à air. Le poids par unité de surface du dépôt est contrôlé. La formation du revêtement se fait alors par chauffage de ce dépôt initial jusqu'à température élevée. 
Le procédé «slurry»a été utilisé par Sylvania [5] pour la réalisation de couches protectrices de siliciure allié de chrome-titane. Ces revêtements sont formés à partir de mélanges de poudres de chrome, de titane et de silicium. Ce procédé, que nous avons étudié, implique une réaction entre les constituants qui a lieu lors de la montée en température avant ou en même temps que la réaction avec le substrat. Notre procédé actuel diffère du procédé Sylvania par l'emploi d'une poudre homogène qui est un siliciure de chrome-titane.

La déposition directe de siliciure simplifie le procédé de fabrication pour les raisons suivantes:

- la poudre a une composition homogène et un point de fusion défini, saire,

- seulement une simple fusion du dépôt est néces-

- le dégagement de chaleur lors de la réaction $\mathrm{du}$ siliciure en présence du substrat est faible,

- par conséquent, le déroulement de la formation du revêtement est plus facile à maîtriser.

Les détails relatifs à la formation du dépôt initial sont présentés dans le tableau III.

Composition de la couche. - La résistance à l'oxydation des siliciures de niobium purs est faible. Alliés au chrome et au titane, ils sont plus résistants à l'oxydation à haute température et, de plus, peu sensibles à l'oxydation à température moyenne (phénomène de la peste) [3].

La composition de siliciure habituellement choisie correspond à $\left(\mathrm{Cr}_{60} \mathrm{Ti}_{40}\right) \mathrm{Si}_{200}$ pour les couches qui sont ensuite imprégnées de $\mathrm{Sn}-\mathrm{Al}$ et à $\left(\mathrm{Cr}_{90} \mathrm{Fe}_{10}\right) \mathrm{Si}_{200}$ pour les couches utilisées sans $\mathrm{Sn}-\mathrm{Al}$.
2.1.2 Formation de la couche de siliciure par fusion. - Principe du procédé. - Les échantillons recouverts de poudre de siliciure sont soumis à un traitement thermique sous vide poussé. Le dépôt de poudre de siliciure de chrome-titane est porté au-dessus de son point de fusion. Au moment de la fusion du siliciure, le dépôt réagit avec le substrá et se solidifie en formant une couche de siliciure is $\mathrm{Cr}, \mathrm{Ti}, \mathrm{Nb}$ (Fig. 7).

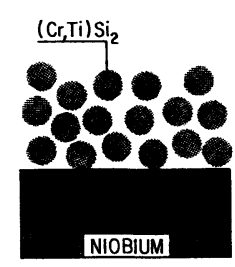

DEPOT DE SILICIURE

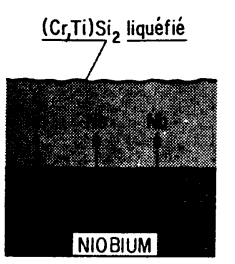

FUSION ETREACTION $1300^{\circ} \mathrm{C}$

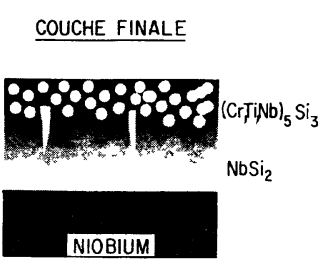

SOLIDIFICATION
FIg. 7. - Principe de la formation de la couche de siliciure $\mathrm{Cr}, \mathrm{Ti}, \mathrm{Nb}$.

Programme de chauffage. - Le programme de chauffage (Fig. 8) est caractérisé par une montée en température de deux heures et demie jusqu'à la température maximale de $1500^{\circ} \mathrm{C}$ environ. Cette température est maintenue pendant une heure avant le refroidissement.

La montée lente en température permet l'élimination des constituants volatils du liant organique avant le moment critique où la couche se forme par fusion. Le recuit a pour but d'homogénéiser la couche de siliciure.

\title{
TABLEAU III
}

\section{Préparation du Slurry}

Granulométrie des poudres de siliciure :

Liant :

Rapport en masse poudre/liant :

$<45 \mu$

Projection par pistolet à air

laque nitrocellulosique

$1 / 1$

Composition des poudres de siliciure utilisées (en fractions atomiques)

\author{
$\left(\mathrm{Cr}_{60} \mathrm{Ti}_{40}\right) \mathrm{Si}_{200}$ (composition standard) \\ $\left(\mathrm{Cr}_{\mathbf{8 0}} \mathrm{Ti}_{20}\right) \mathrm{Si}_{200}$ \\ $\left(\mathrm{Cr}_{45} \mathrm{Ti}_{55}\right) \mathrm{Si}_{200}$
}

$$
\begin{aligned}
& \left(\mathrm{Cr}_{60} \mathrm{Ti}_{40}\right) \mathrm{Si}_{170} \\
& \left(\mathrm{Cr}_{60} \mathrm{Ti}_{40}\right) \mathrm{Si}_{210} \\
& \left(\mathrm{Cr}_{90} \mathrm{Ti}_{40}\right) \mathrm{Si}_{400}
\end{aligned}
$$

Vitesses de montée en température entre 1200 et $1400^{\circ} \mathrm{C}$ (voir Fig. 8)
a) Chauffage très rapide :
$200^{\circ} \mathrm{C} / \mathrm{min}$
b) Chauffage normal :
$10^{\circ} \mathrm{C} / \mathrm{min}$ (vitesse standard)
c) Chauffage lent : 
On a étudié l'influence de différentes vitesses de chauffage dans le domaine de températures allant de 1200 à $1400^{\circ} \mathrm{C}$ sur la formation de la couche (Fig. 8 et tableau III).

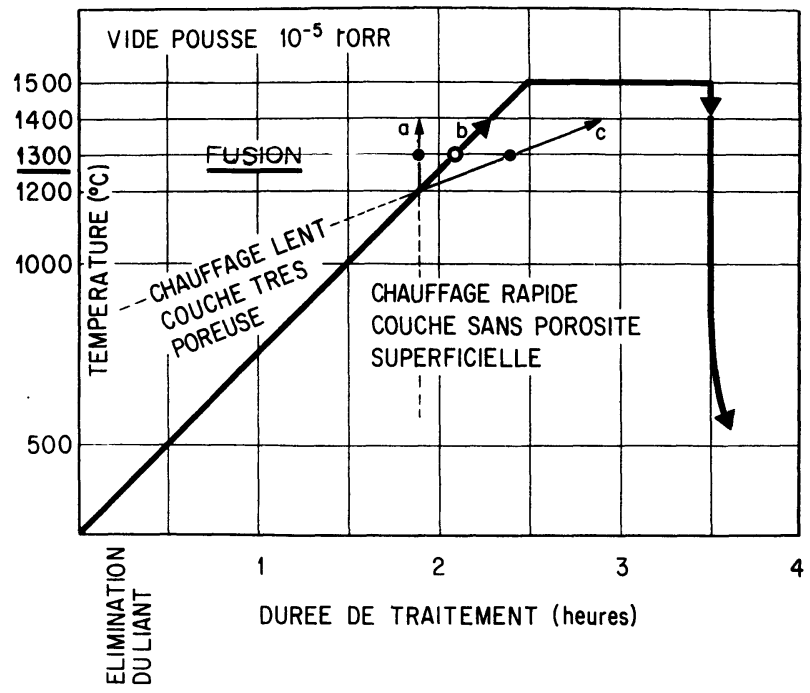

FIG. 8. - Programme de chauffage.

Influence de différents paramètres sur la structure $d u$ revêtement. - L'influence de différents facteurs sur la structure du revêtement est étudiée en faisant varier :

- la composition de la poudre de siliciure, et

- le programme de chauffage, en particulier la vitesse de montée en température, au moment précis où l'on passe la température de fusion du dépôt.
L'influence de la composition de la poudre de siliciure a été étudiée en faisant varier le rapport chrometitane et la teneur en silicium entre certaines limites indiquées dans le tableau III.

L'examen métallographique n'a pas permis d'observer une influence très sensible de la composition sur la structure du revêtement.

En revanche, c'est la cinétique de chauffage qui influe sur la porosité de la couche.

Un chauffage très rapide $\left(200^{\circ} \mathrm{C} / \mathrm{min}\right)$ entraîne la formation de couches compactes non poreuses. En cas de chauffage lent $\left(3,3-10^{\circ} \mathrm{C} / \mathrm{min}\right)$ la zone extérieure de la couche devient poreuse. Pour obtenir un degré de porosité constant, il faut maintenir constant, outre la vitesse de chauffage, le poids du dépôt initial.

2.1.3 Aspect et composition des couches de siliciure formées sur différents substrats. - Des couches de siliciure, formées à partir de poudre de siliciure de chrome-titane ont été déposées sur du niobium pur, sur différents alliages binaires et sur des alliages à résistance mécanique élevée de composition complexe (tableau IV).

Ces revêtements offrent un aspect très semblable dans leur structure et dans leur composition de base.

La structure des couches est caractérisée par une séquence de zones de siliciure de compositions différentes, ainsi que le montrent les figures $9 a, 9 b$ (revêtements sur du niobium pur et sur l'alliage P353M).

L'analyse à la microsonde (Fig. 9b, 10b) révèle pour la couche sur niobium pur :

- une zone intérieure adhérente au substrat et de

TABleaU IV

Spécification pour différents matériaux de base utilisés comme substrat

\begin{tabular}{|c|c|c|c|}
\hline Mise en solution & $\begin{array}{c}\text { Teneur en carbone } \\
(\% \text {-masse })\end{array}$ & $\begin{array}{l}\text { Forme } \\
\text { Epaisseur } \\
\text { Diamètre }\end{array}$ & $\begin{array}{l}\text { Dureté Vickers } \\
(5 \mathrm{~kg}) \text { à tempé- } \\
\text { rature ambiante }\end{array}$ \\
\hline
\end{tabular}

\begin{tabular}{|c|c|c|c|c|}
\hline \multicolumn{3}{|c|}{ Alliage P353M : Nb-10 W-3,8 Zr-1,2 Ti-2,8 V-C ( $\%$-masse) } & $(\mathrm{mm})$ & $\left(\mathrm{kg} / \mathrm{mm}^{2}\right)$ \\
\hline CA 132 & $1 \mathrm{~h}, 1800^{\circ} \mathrm{C}$ & $0,32-0,35$ & tôle, 3 & 205 \\
\hline CA 225 & & & tôle, 2,5 & 190 \\
\hline CA 249 & $1 \mathrm{~h}, 2000^{\circ} \mathrm{C}$ & $0,30-0,31$ & tôle, 2,3 & 220 \\
\hline & & $0,46-0,47$ & tôle, 4 & 190 \\
\hline & $1 \mathrm{~h}, 1800^{\circ} \mathrm{C}$ & $0,39-0,41$ & barre, 18 & 270 \\
\hline & $1 \mathrm{~h}, 2000^{\circ} \mathrm{C}$ & & barre, 18 & 260 \\
\hline \multicolumn{3}{|c|}{ Alliage $\mathrm{Cb}-752: \mathrm{Nb}-10 \mathrm{~W}-2,5 \mathrm{Zr}(\%$-masse $)$} & barre, 12 & 190 \\
\hline \multicolumn{3}{|c|}{ Alliage B-66 : Nb-5 Mo-5 V-1 Zr ( $\%$ masse) } & tôle, 2 & 220 \\
\hline \multicolumn{5}{|c|}{ Alliages binaires } \\
\hline \multicolumn{3}{|l|}{ Nb-15 Ti (at.- \%) } & tôle, 2 & 155 \\
\hline \multicolumn{3}{|l|}{$\mathrm{Nb}-5 \mathrm{Zr}$} & tôle, 2 & 140 \\
\hline \multicolumn{3}{|l|}{$\mathrm{Nb}-5 \mathrm{Zr}-\mathrm{C}$} & tôle, 2 & 140 \\
\hline \multicolumn{3}{|l|}{$\mathrm{Nb}-15 \mathrm{~V}$} & tôle, 2 & 150 \\
\hline \multicolumn{3}{|l|}{$\mathrm{Nb}-5 \mathrm{~W}$} & tôle, 2 & 145 \\
\hline
\end{tabular}




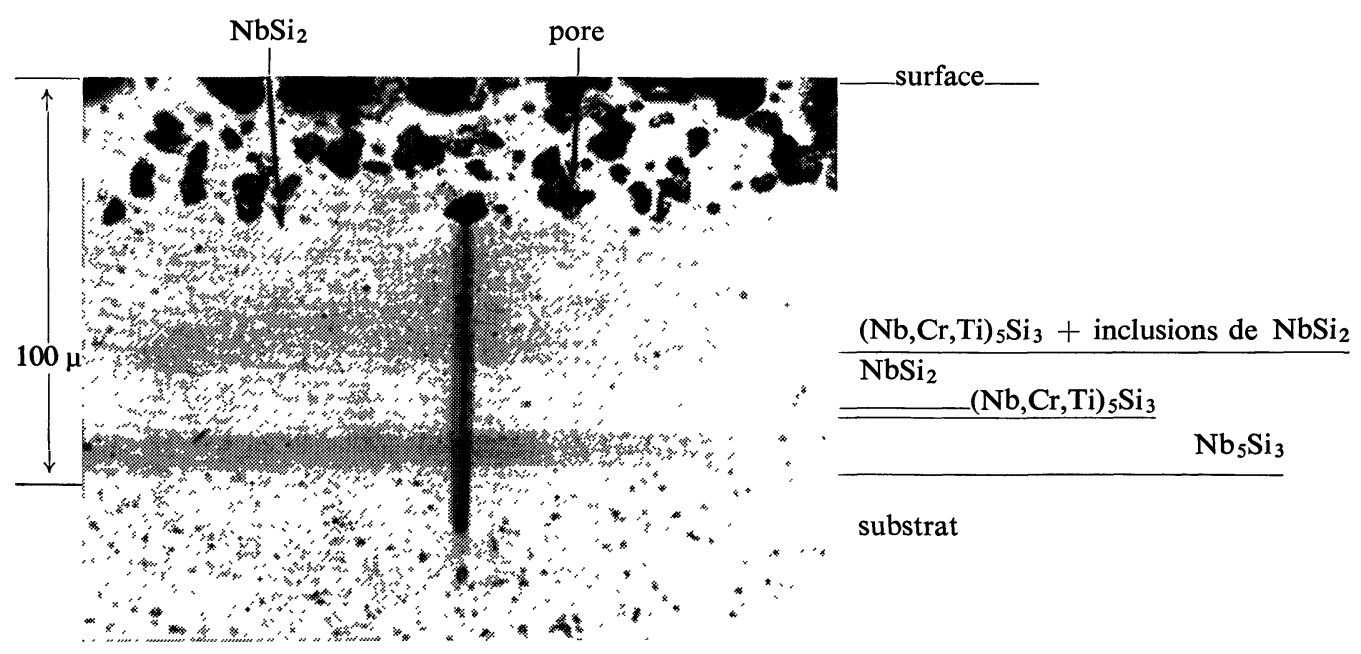

(a)

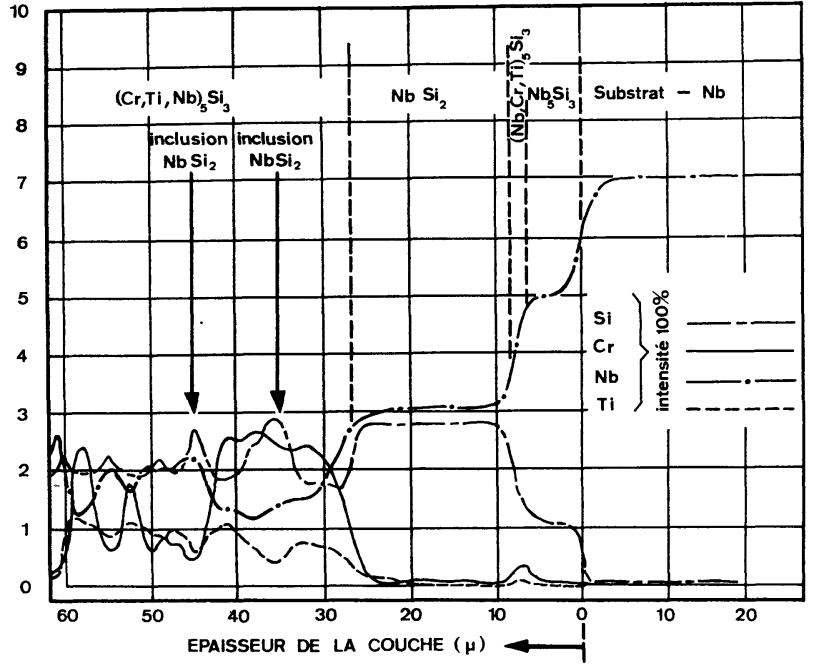

(b)

FIG. 9. - Couche de siliciure sur niobium pur.

9a) Micrographie.

Vitesse de chauffage $10{ }^{\circ} \mathrm{C} / \mathrm{min}$

Substrat : niobium

Poudre : $\left(\mathrm{Cr}_{60}, \mathrm{Ti}_{40}\right) \mathrm{Si}_{200}$

9b) Microsonde.

composition $\mathrm{Nb}_{5} \mathrm{Si}_{3}$. Elle contient des traces de chrome et de titane,

- une zone intermédiaire de composition $\mathrm{NbSi}_{2}$, qui ne contient pas ces deux éléments,

- une zone extérieure, enrichie en chrome et en titane, de composition $(\mathrm{Cr}, \mathrm{Ti}, \mathrm{Nb})_{5} \mathrm{Si}_{3}$. Cette zone contient souvent des inclusions de $\mathrm{NbSi}_{2}$ comme deuxième phase.

Dans le cas d'un substrat en alliage de niobium, ces mêmes zones contiennent des éléments additionnels, qui sont les éléments d'alliage du substrat ; leur teneur diminue à mesure qu'on s'éloigne du substrat.

Une nette division de la zone intérieure de composition $\mathrm{Nb}_{5} \mathrm{Si}_{3}$ en deux phases distinctes apparaît ici, ce qui ne se produit pas dans les couches déposées sur du niobium pur. Des traces de chrome et de titane se trouvent dans la phase la plus éloignée du substrat.

La zone $\mathrm{NbSi}_{2}$ contient parfois de fines inclusions d'une deuxième phase qui, par sa composition, pourrait correspondre au siliciure $(\mathrm{Cr}, \mathrm{Ti}, \mathrm{Nb})_{5} \mathrm{Si}_{3}$ de la zone extérieure.

La figure 11 montre quelques échantillons de niobium et d'alliages de niobium recouverts de siliciure.

2.1.4 Imperfections structurales des couches de siliciure. - Après fabrication, les couches de siliciure présentent des imperfections localisées qui peuvent diminuer l'efficacité du revêtement. Ces imperfections sont réparées en grande partie par l'action de l'étain-aluminium pendant l'imprégnation qui sera décrite par la suite.

Des défauts ou anomalies du revêtement peuvent apparaître :

- au niveau de l'interface substrat-couche (manque d'adhérence de la couche),

- à l'intérieur de la couche (cavités dans la couche),

- en surface (irrégularités de la surface).

Les investigations relatives aux origines de ces défauts, dont les figures $12 a, b, c$ donnent des exemples, ont conduit aux résultats suivants :

Adhérence. - Des fissures locales à l'intérieur du revêtement, parallèles à l'interface substrat-couche, peuvent diminuer l'adhérence du revêtement (Fig. 12a). Ce genre de défauts, qui n'a pas été observé sur du niobium pur après formation de la couche, apparaît de préférence dans les couches déposées sur des alliages à résistance élevée $(\mathrm{P} 353 \mathrm{M}, \mathrm{Cb} 752)$ au niveau de la zone $\mathrm{Nb}_{5} \mathrm{Si}_{3}$.

La présence dans $\mathrm{Nb}_{5} \mathrm{Si}_{3}$ d'éléments d'alliage provenant du substrat, ou simplement des tensions de dilatation accrues de la couche de siliciure, en présence d'un substrat de plus grande résistance mécanique, peut être à l'origine de ces défauts.

Cavités à l'intérieur de la couche. - Ces défauts sont apparus, quelles que furent les conditions de fabrica- 


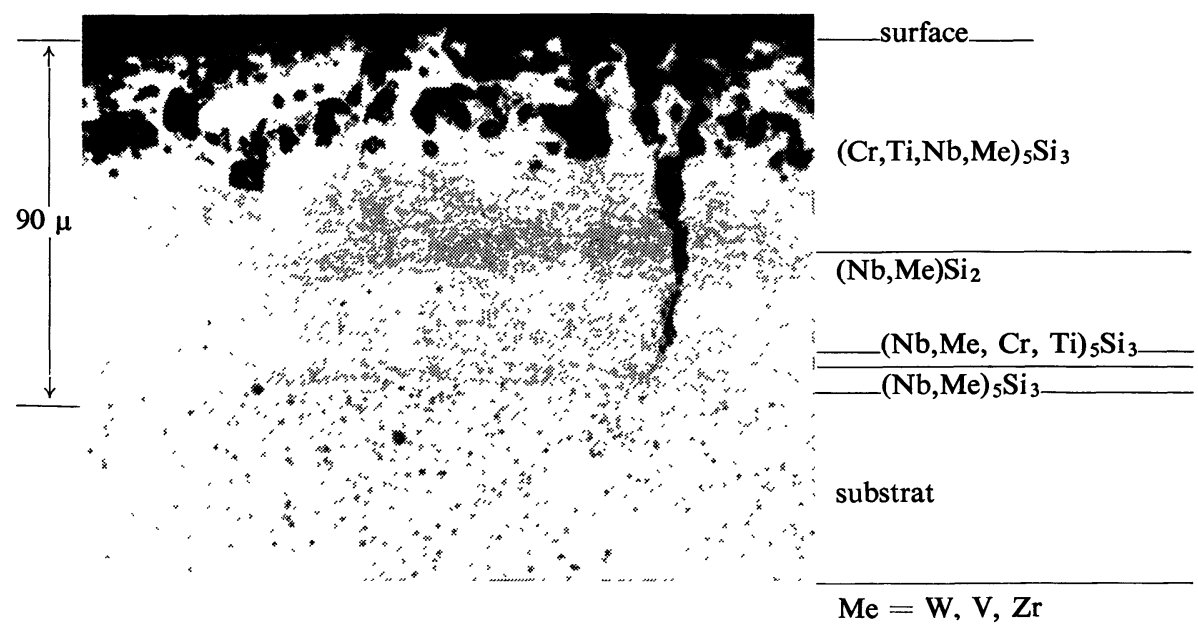

(a)

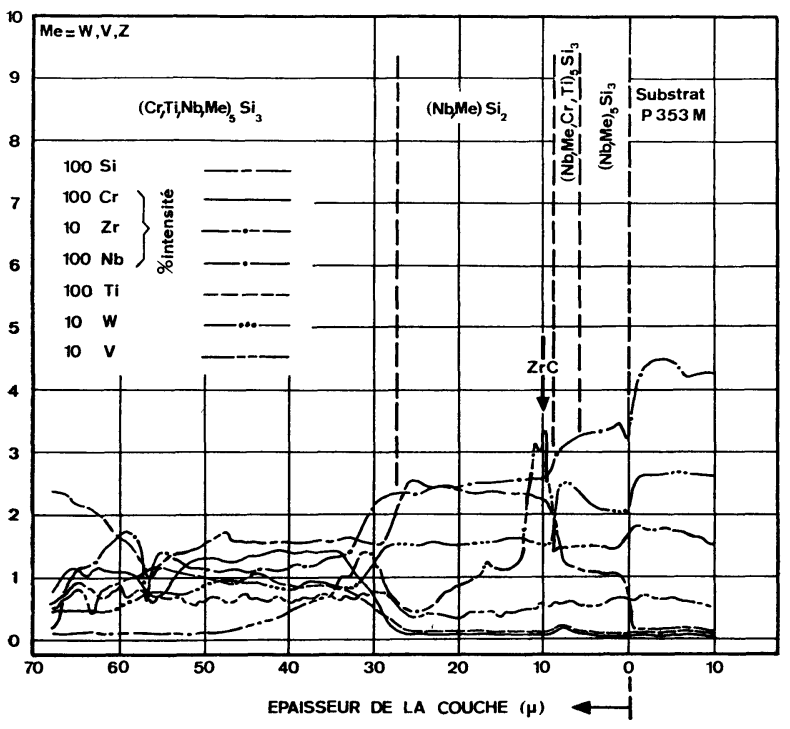

(b)

FIg. 10. - Couche de siliciure sur alliage P $353 \mathrm{M}$.

10a) Micrographie.

Vitesse de chauffage $10^{\circ} \mathrm{C} / \mathrm{min}$

Substrat : P353M.

Poudre : $\left(\mathrm{Cr}_{60}, \mathrm{Ti}_{40}\right) \mathrm{Si}_{200}$.

10b) Microsonde.

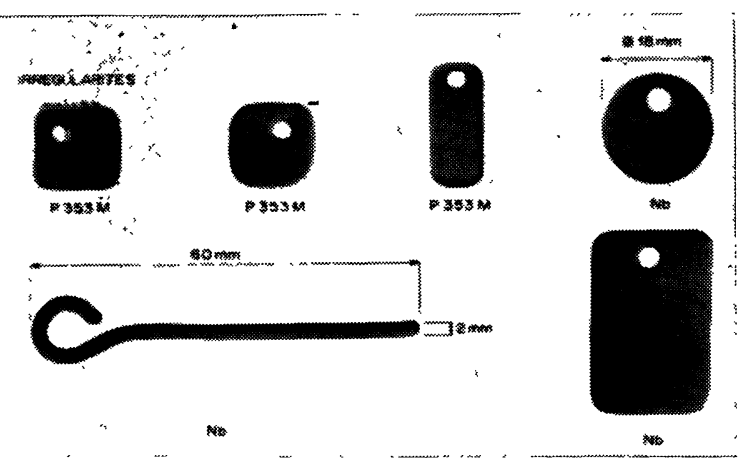

FIG. 11. - Différents échantillons recouverts de siliciure $\mathrm{Cr}, \mathrm{Ti}, \mathrm{Nb}$. tion (Fig. 12b) ; nous pensons qu'il s'agit de retassures qui sont liées à la solidification du dépôt.

Irrégularités de la surface. - Des différences locales de température entre différents endroits de l'échantillon, au moment où l'on atteint le point de fusion $\mathrm{du}$ dépôt de siliciure, peuvent provoquer une mauvaise synchronisation de la formation du revêtement. $\mathrm{Ce}$ fait peut favoriser l'apparition d'irrégularités de la couche, qui apparaissent sous forme de protubérances (Fig. 12c) locales ou de surépaisseurs inhabituelles dans certaines parties de l'échantillon. Les couches déposées sur des alliages de niobium (P353M, Cb752) à teneur élevée en éléments d'alliage, sont particulièrement sensibles à ce genre de défaut.

L'utilisation d'une poudre de siliciure de composition $\left(\mathrm{Cr}_{60} \mathrm{Ti}_{40}\right) \mathrm{Si}_{170}$, à teneur en silicium réduite, semble diminuer la tendance à l'apparition d'irrégularités de la surface du siliciure.

Notre méthode de préparation ne permet cependant pas d'éviter à coup sûr l'apparition de ce type de défauts.

2.1.5 Discussion de la formation de la couche de siliciure. - Les phénomènes observés lors de la formation des couches et l'aspect de leur structure finale laissent supposer qu'elles se forment de la manière suivante :

- Au moment de sa fusion, le dépôt de siliciure de chrome-titane dissout du niobium du substrat ainsi que des éléments d'alliage éventuellement présents.

- Le siliciure liquide s'enrichit donc en niobium et se solidifie en formant deux composés différents : $\mathrm{NbSi}_{2}$ et $(\mathrm{Cr}, \mathrm{Ti}, \mathrm{Nb})_{5} \mathrm{Si}_{3}$.

L'augmentation de sa teneur en niobium élève le point de fusion du siliciure. La transformation du siliciure fondu en couche solide correspond à un déplacement isotherme de concentration le long de la ligne $(\mathrm{Cr}, \mathrm{Ti}) \mathrm{Si}_{2} \rightarrow \mathrm{Nb}$ dans le diagramme de phase quasi ternaire (Cr,Ti)-Si-Nb. 


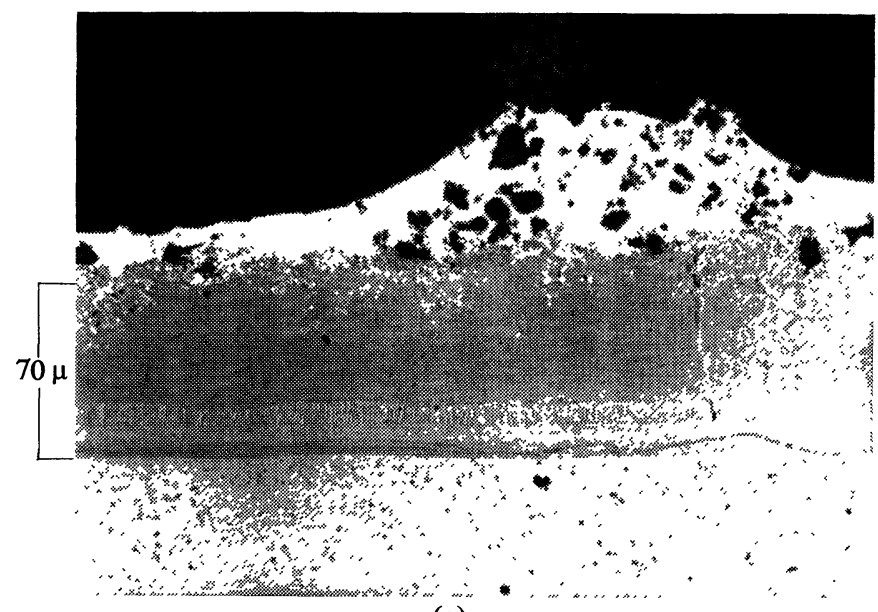

(a)

Deux essais de trempe de la couche pendant sa formation ont été effectués et semblent confirmer cette hypothèse. Les résultats sont présentés dans les figures $13 a, b$. Dans le premier cas, on observe une couche de siliciure uniforme avec des teneurs constantes en chrome, titane et niobium, ce qui correspond au premier stade de dissolution du niobium. Dans le deuxième cas, où la transformation était plus avancée, on remarque déjà une zone plus importante de $\mathrm{NbSi}_{2}$ et des inclusions de $\mathrm{NbSi}_{2}$ dans le $(\mathrm{Cr}, \mathrm{Ti}$, $\mathrm{Nb})_{5} \mathrm{Si}_{3}$.

Il est possible que $\mathrm{NbSi}_{2}$, dont le point de fusion est d'environ $2000^{\circ} \mathrm{C}$, précipite comme phase primaire, ce qui n'exclurait cependant pas la croissance finale de la zone intérieure de $\mathrm{NbSi}_{2}$ par diffusion à

Substrat : P353M

(revêtement imprégné)

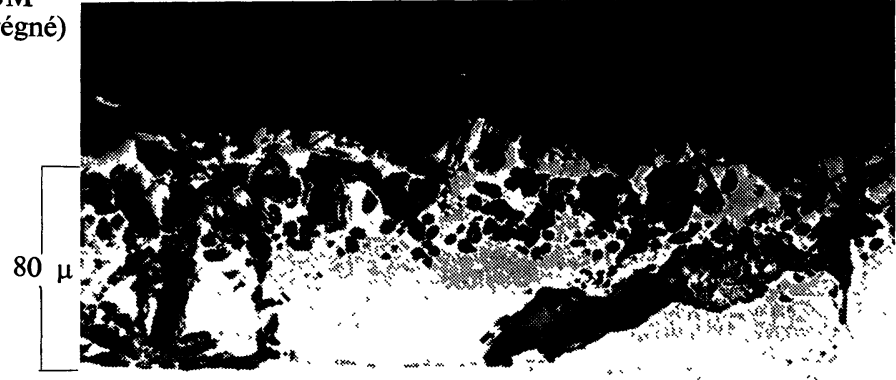

Substrat : P353M

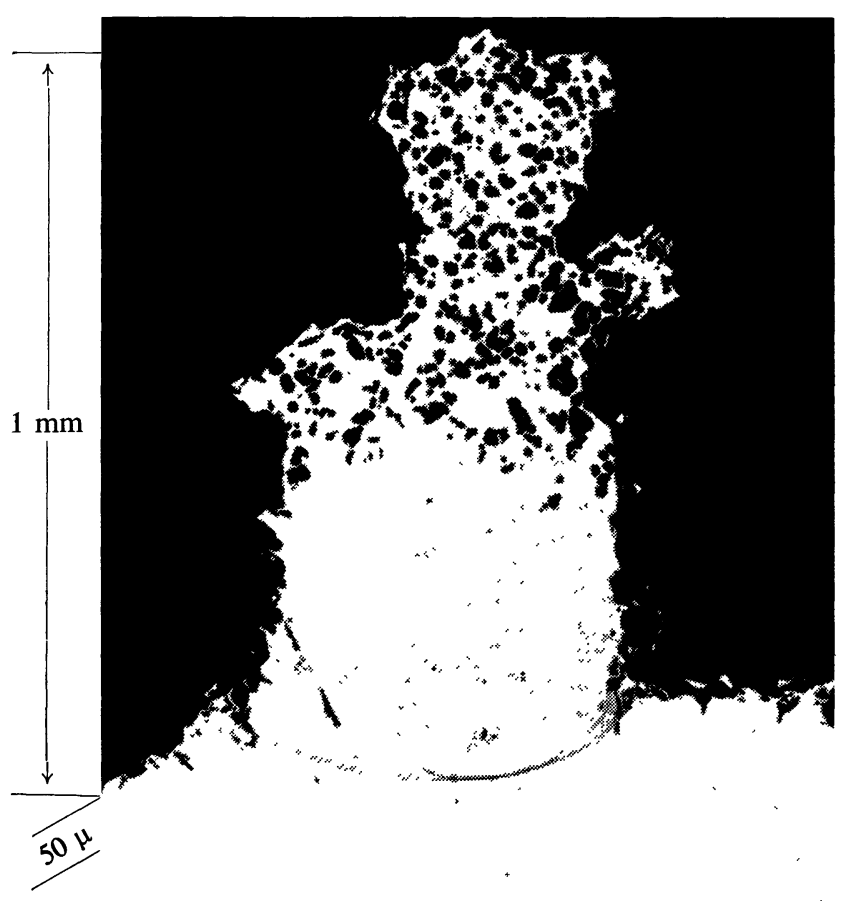

(c)

Fig. 12. - Défauts de la couche de siliciure.

12a) Fissures à l'intérieur.

12b) Cavités de la couche.

12c) Irrégularités en surface. (b)

l'état solide. La croissance de la zone de $\mathrm{Nb}_{5} \mathrm{Si}_{3}$ se fait par diffusion à l'état solide.

En général, on obtient une couche finale très régulière grâce à la répartition du dépôt au moment de sa fusion. Le fait que le liqưide ne s'écoule pas laisse supposer que la formation du revêtement a lieu dans un temps très court.

Le fait que le degré de porosité superficielle de la couche de siliciure est fonction de la vitesse de chauffage est encore difficile à interpréter. Toutefois, il est important de bien contrôler le degré de porosité de la couche de siliciure par des vitesses constantes pour obtenir une imprégnation correcte du siliciure par l'alliage Sn-Al.

2.2 Imprégnation de la couche de siliciure par l'étain-aluminium. - L'imprégnation de la zone extérieure de la couche de siliciure se fait par traitement dans des bains de $\mathrm{Sn}-\mathrm{Al}$ dans les conditions indiquées dans le tableau V.

Il existe deux manières de procéder (Fig. 14) :

a) par simple remplissage des pores lorsqu'il s'agit d'une couche à porosité superficielle ouverte,

b) par pénétration de l'étain-aluminium dans les joints de grains dans le cas d'une couche initialement compacte.

Sous l'action prolongée de Sn-Al liquide, la zone superficielle de la couche de siliciure subit des trans- 


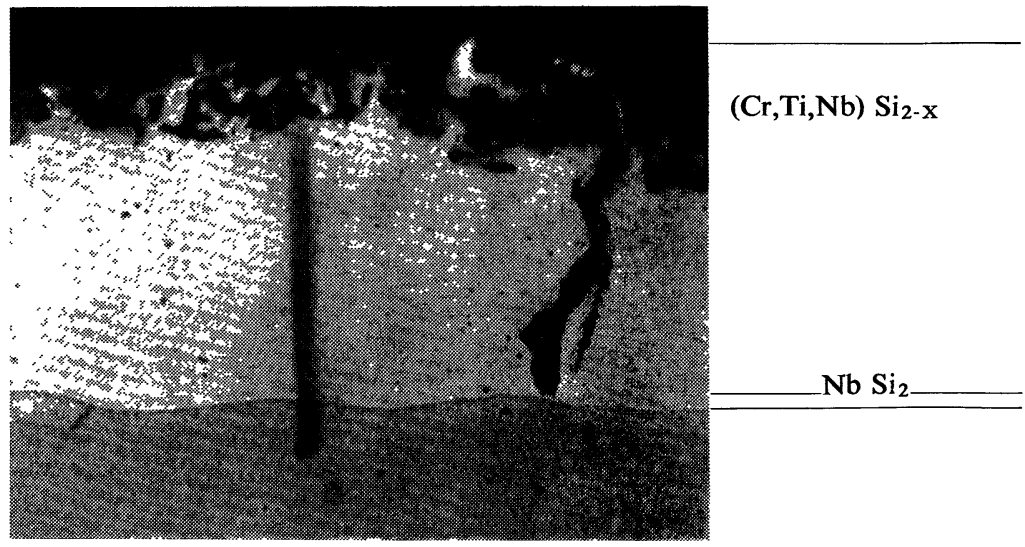

(a)

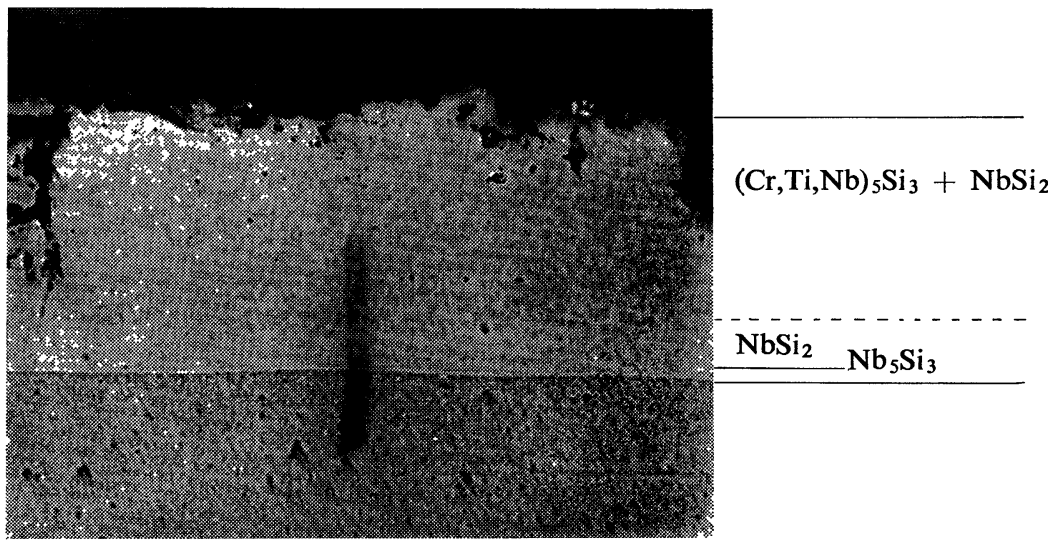

(b)

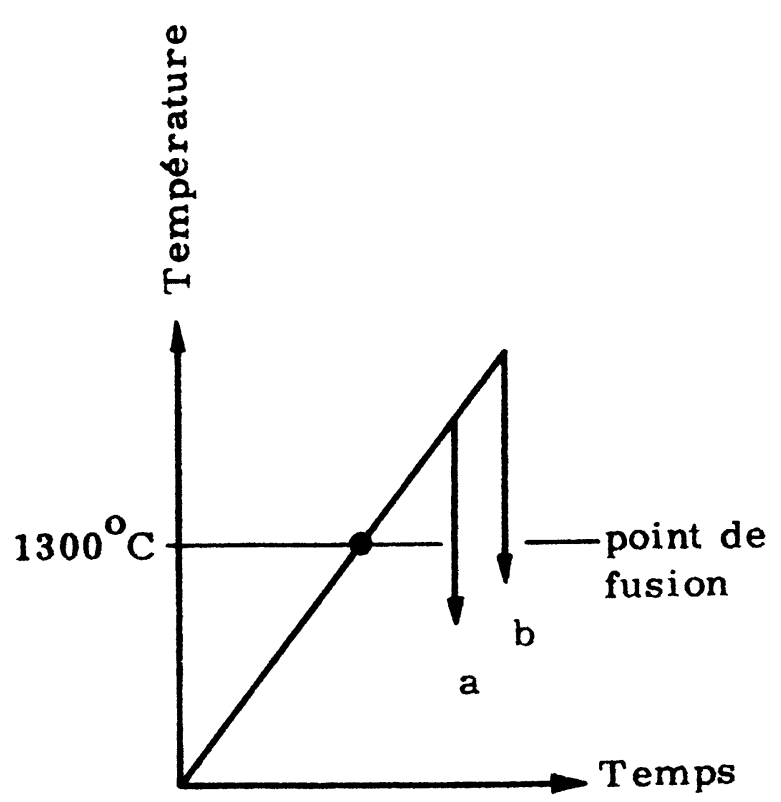

FIG. 13. - Structure de couches de siliciure refroidies pendant leur formation.

\section{TABLEAU $\mathbf{V}$}

Conditions de traitement des couches de siliciure dans le bain de $\mathrm{Sn}-\mathrm{Al}$

Bains : $\quad$ Alliages de $\mathrm{Sn}-\mathrm{Al}$, saturés de silicium

Creusets : $\quad \mathrm{Al}_{2} \mathrm{O}_{3}$

Atmosphère : vide, $10^{-2}$ Torr.

[1] Imprégnation des couches de siliciure poreuses en surface : $90 \mathrm{Sn} / 10 \mathrm{Al} / \mathrm{Si}, 900^{\circ} \mathrm{C}, 1 \mathrm{~h}$.

[2] Imprégnation des couches de siliciure compactes sans porosité : $90 \mathrm{Sn} / 10 \mathrm{Al} / \mathrm{Si}, 1200^{\circ} \mathrm{C}, 20 \mathrm{~min}$.

formations progressives; on distingue trois stades consécutifs (Fig. 15) :

1) remplissage des pores et des fissures du siliciure par Sn-Al,

2) attaque intergranulaire du siliciure par $\mathrm{Sn}-\mathrm{Al}$, ce qui se traduit par

- une augmentation de la porosité d'une couche initialement poreuse, 


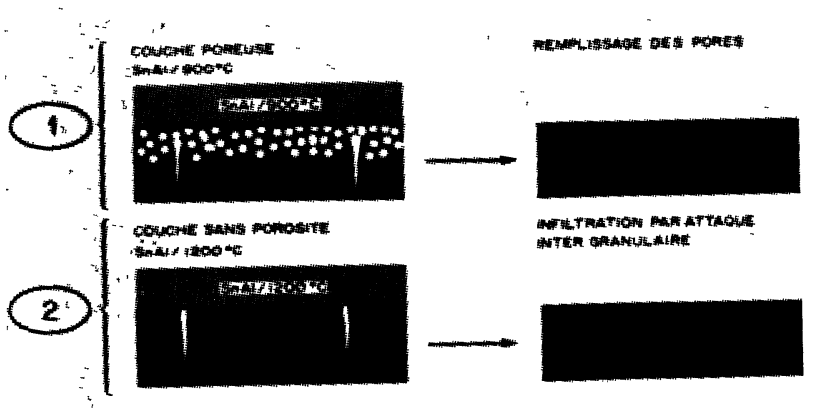

FIG. 14. - Types d'imprégnation des couches de siliciure.
- une pénétration de $\mathrm{Sn}-\mathrm{Al}$ dans la zone superficielle d'une couche initialement compacte.

3) désagrégation de la surface de la couche par l'isolation des grains de siliciure due à l'attaque intergranulaire prolongée.

Afin d'obtenir une imprégnation complète sans toutefois provoquer la dissolution du siliciure dans le bain, les conditions optimales de trempage sont choisies en fonction de la structure et de la porosité initiales du siliciure. L'infiltration des couches compactes requiert, par exemple, une température ou une durée

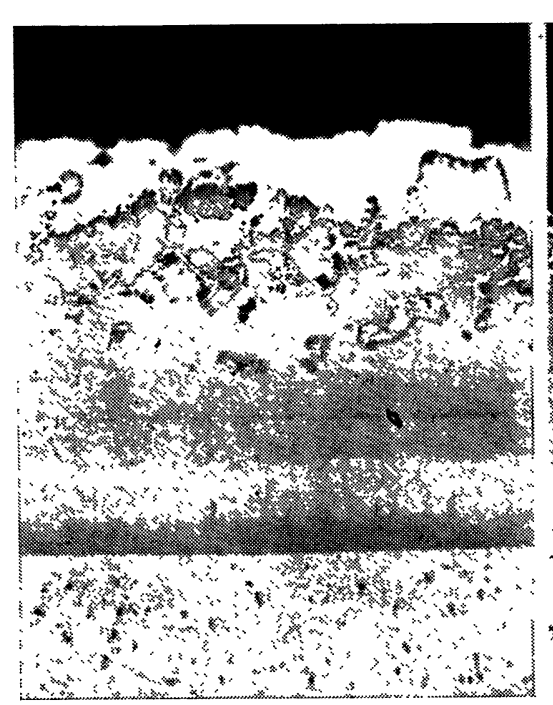

(a)

Durée du traitement : $1 \mathrm{~h}$ remplissage des pores Substrat : niobium Couche de siliciure initialement poreuse Imprégnation : $90 \mathrm{Sn} / 10 \mathrm{~A} 1 / \mathrm{Si}, 900{ }^{\circ} \mathrm{C}$

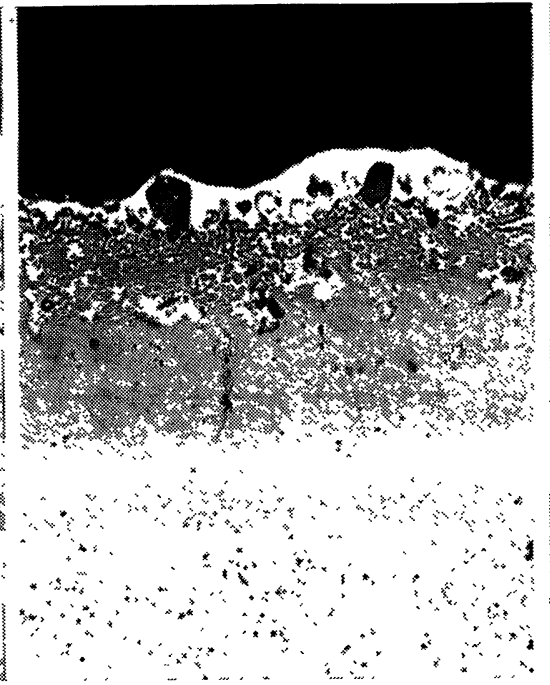

(b)

$2 \mathrm{~h}$

début de l'attaque intergrabinaire par Sn-Al

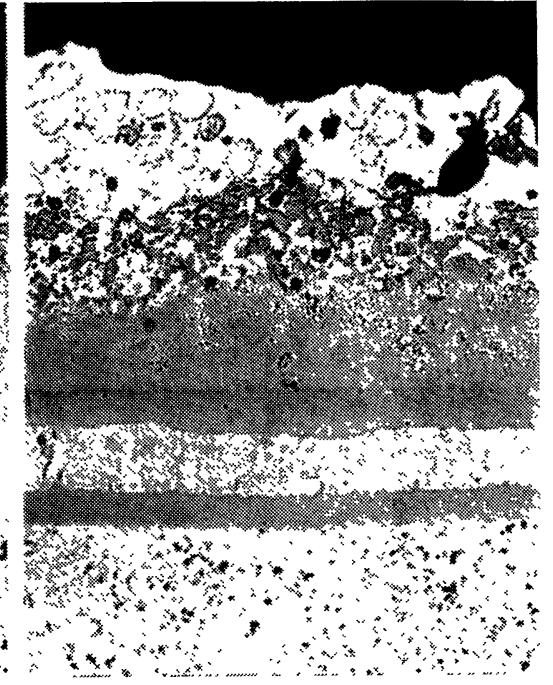

(c)

$5 \mathrm{~h}$

isolation des grains de siliciure de la zone superficielle

FIG. 15. - Différents stades d'imprégnation.

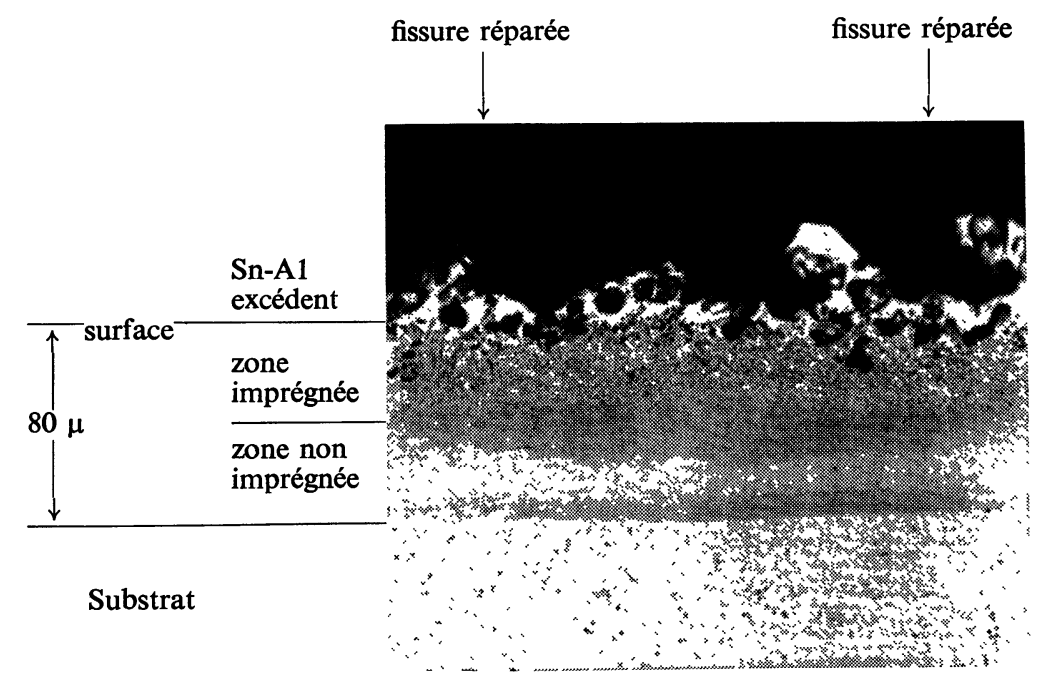

Substrat : Alliage P353M

Couche de siliciure : chauffage rapide

Imprégnation : $90 \mathrm{Sn} / 10 \mathrm{Al} / \mathrm{Si}, 1200{ }^{\circ} \mathrm{C}, 20 \mathrm{~min}$

FIG. 16. - Couche de siliciure initialement compacte, imprégnée par voie intergranulaire. 
de traitement supérieures à celles utilisées pour les couches initialement poreuses, et conduit généralement à une porosité fine.

Pendant l'imprégnation, l'étain-aluminium liquide exerce un effet de guérison sur les fissures et sur les autres défauts présents dans la couche de siliciure après sa fabrication (Fig. 17a, b).

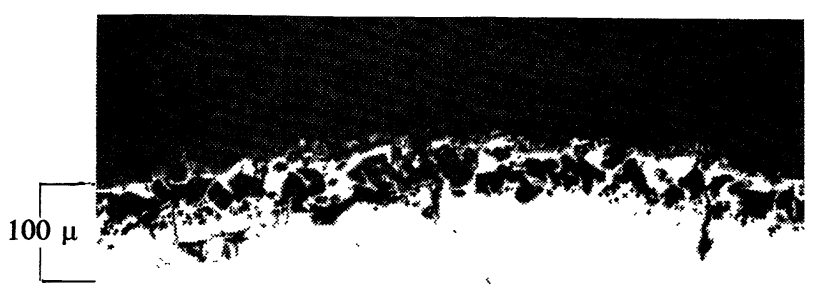

(a)

avant imprégnation

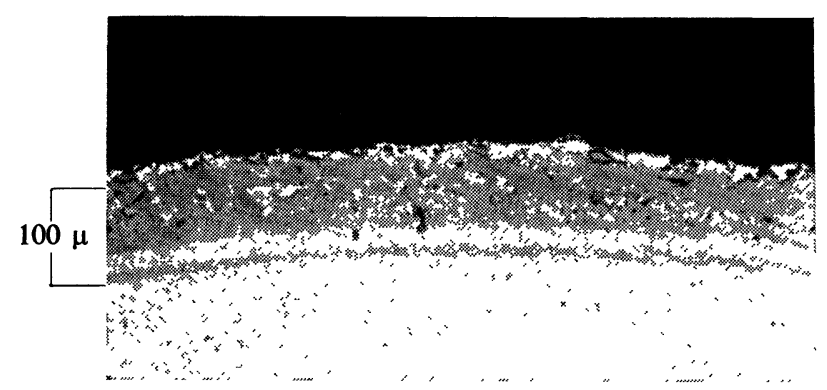

Substrat : niobium

Couche de siliciure initialement poreuse.

Imprégnation : $90 \mathrm{Sn} / 10 \mathrm{Al} / \mathrm{Si}, 900^{\circ} \mathrm{C}, 1 \mathrm{~h}$.

après imprégnation

Fig. 17. - Couche de siliciure avant et après imprégnation.

Ce phénomène est dû à la solubilité du siliciure dans le $\mathrm{Sn}$-Al liquide qui assure ainsi le transport et la diffusion rapide du siliciure à l'intérieur de la couche $\left({ }^{4}\right)$. L'action de la phase liquide se manifeste par le comblement des fissures de la zone intérieure compacte et par une recristallisation de la zone extérieure poreuse.

L'effet de guérison ne s'étend qu'aux couches de siliciure qui ont bénéficié d'une imprégnation complète par l'étain-aluminium liquide.

Après le traitement, l'imprégnation demeure parfois imparfaite dans certains endroits de la couche par suite d'un mouillage défectueux. De tels cas sont observés plus fréquemment dans le cas de couches sur alliage P $353 \mathrm{M}$ que dans celui de couches sur niobium pur. Ces difficultés de mouillage sont probablement liées à la présence d'un film de $\mathrm{Al}_{2} \mathrm{O}_{3}$ qui empêche le contact entre le siliciure et $\mathrm{Sn}-\mathrm{Al}$ liquide.

Certaines dispersions dans la durée de vie pendant l'oxydation peuvent s'expliquer par une imprégnation incomplète.

3. ENDURANCE DU REVÊTEMENT AUX TESTS D'OXYDATION. - L'endurance des couches protectrices à base

(4) Des phénomènes semblables sont connus dans le frittage en présence d'une phase liquide. de composés intermétalliques dépend fortement des conditions particulières d'oxydation. Le revêtement de siliciure $\mathrm{Cr}, \mathrm{Ti}, \mathrm{Nb} / \mathrm{Sn}-\mathrm{Al}$ ne fait pas exception à cette règle.

Nous avons effectué les tests d'oxydation à l'air selon différents programmes de chauffage (tableau II), afin de contrôler les comportements du revêtement dans des conditions variées.

Les résultats d'oxydation obtenus jusqu'à ce jour sont indiqués dans le tableau II et illustrés dans les figures 3 et 4 . A titre de comparaison, nous avons également représenté les durées de vie d'autres systèmes de protection que nous avons étudiés.

Les résultats d'oxydation indiquent que la durée de vie est fonction des facteurs suivants :

- température maximale,

- nombre de refroidissements qui interrompent le séjour à haute température,

- vitesse de refroidissement et de réchauffage.

3.1 Test d'oxydation de longue durée. - Les résultats obtenus dans les divers tests sont les suivants :

Test A : Une oxydation à température constante donne des durées de vie maximales supérieures à $600 \mathrm{~h}$ à $1100^{\circ} \mathrm{C}$ et à $300 \mathrm{~h}$ à $1200^{\circ} \mathrm{C}$.

Dans le cas de tests d'oxydation cycliques, la durée de protection est, de manière générale, réduite par rapport à l'oxydation à température constante.

Test B : Les changements de températures très rapides influent peu sur la durabilité des revêtements. On observe, par exemple, une durée de vie de plus de $1000 \mathrm{~h}$ à $1100^{\circ} \mathrm{C}$, bien que le traitement comprenne 900 cycles [6].

Une diminution des vitesses de chauffage et de refroidissement entraîne une sensible réduction de la durabilité.

Test $\mathrm{C}$ : Dans ce test cyclique où la température maximale est de $1200^{\circ} \mathrm{C}$, ces vitesses sont de $80^{\circ} \mathrm{C} / \mathrm{min}$.

Les valeurs les plus fréquentes de la durée de vie se situent entre 100 et 150 cycles (Fig. 3), ce qui correspond à environ 30 à $50 \%$ de la durée observée dans le test statique à $1200^{\circ} \mathrm{C}$.

Test E : Dans ce test (Fig. 4), qui comprend une montée en température extrêmement lente $\left(5^{\circ} \mathrm{C} / \mathrm{min}\right)$, un palier de $10 \mathrm{~min}$ suivi d'un refroidissement très rapide, la durée de protection est restreinte. Les revêtements qui ont bien résisté dans les tests à cycles plus rapides $(B, C)$ cèdent pour la plupart après 10 à 40 cycles, ce qui correspond à un séjour total de moins de $10 \mathrm{~h}$ à température maximale.

Cependant, certains échantillons supportent exceptionnellement un grand nombre de cycles (plus de 200 cycles). Il n'a pas été possible de mettre en évidence une influence nette du mode de fabrication ou de la porosité sur l'endurance du revêtement. 
Test D : Si les chauffages lents n'ont lieu qu'entre 700 et $1200^{\circ} \mathrm{C}$, on constate que quelques échantillons qui résisteraient mal dans le test $\mathrm{C}$ montrent une endurance accrue. Cependant, cette observation n'est basée que sur un nombre d'essais encore très limité.

Couches de siliciure non imprégnées. - A titre de comparaison, nous avons testé un nombre limité de couches de siliciure $\mathrm{Cr}, \mathrm{Ti}, \mathrm{Nb}$ non traitées par $\mathrm{Sn}-\mathrm{Al}$. On a noté une durée de vie maximale de 120 cycles pour le test $C$, de 35 cycles pour le test $D$ et de 10 cycles pour le test $\mathrm{E}$. Ces valeurs semblent indiquer que la durée de protection, dans ce cas également, est fonction des vitesses de chauffage et de refroidissement.

3.2 Test de cyclage sans palier : choc thermique. Une sensibilité à des changements lents de température peut se déduire également des résultats de tests cycliques sans palier (tests $\mathrm{F}, \mathrm{G}$, voir tableau II) dans le cas des revêtements de type $\mathrm{Nb}(\mathrm{Ti}) \mathrm{Si}_{2} / \mathrm{Sn}-\mathrm{Al}$.

Test F : Le revêtement de siliciure $\mathrm{Cr}, \mathrm{Ti}, \mathrm{Nb} / \mathrm{Sn}-\mathrm{Al}$ résiste très bien au cyclage rapide. La durabilité dépasse parfois 1000 cycles. Cette bonne résistance au choc thermique a été également confirmée par les tests effectués ailleurs [6] (plus de 600 cycles sans défaillance).

En résumé, la durée de protection dans les tests cycliques dépend fortement des vitesses de chauffage et de refroidissement.

Le revêtement offre une protection durable en dépit des refroidissements fréquents à condition que le passage de la température élevée (test $B$ ) à la température ambiante se fasse rapidement. Des changements de température à une vitesse de l'ordre de $125^{\circ} \mathrm{C} / \mathrm{min}$ influent déjà sensiblement sur la durée de protection dans des cycles d'une heure (test $\mathrm{C}$ ).

Dans sa forme actuelle, le revêtement est vulnéiable à des cyclages extrêmement lents (tests D, E) à température moyenne. La zone des températures la plus critique à cet égard se situe autour de $1000^{\circ} \mathrm{C}$ (test D).

3.3 Test d'impact. - Mentionnons simplement que les impacts, par des particules solides de petite taille arrivant à grande vitesse, n'entraînent pas une défaillance immédiate du revêtement, ce qui semble indiquer une influence favorable de la phase Sn-Al (tableau VI).

3.4 Influence de la composition du substrat sur la durabilité. - Les durées de vie dans différents tests des revêtements déposés sur un alliage de niobium (P353M) sont du même ordre que celles des couches déposées sur du niobium pur.

4. EVOLUTION DU REVÊTEMENT AU COURS DE L'OXYDATION. - Pendant l'oxydation, le revêtement subit des changements de composition et de structure.

\section{TABLEAU VI}

Résultats des tests d'impacts [6]

Projectiles :

- Balle en acier doux, dont une extrémité forme un cône de $90^{\circ}$

$$
\begin{array}{lll}
\text { Poids ............ } & 3 \mathrm{~g} \\
\text { Vitesse d'impact ... } & 260 \mathrm{~m} / \mathrm{s}
\end{array}
$$

- Bille en acier doux

$$
\text { Poids ......... } 0,35 \mathrm{~g}
$$$$
\text { Vitesse d'impact .... } 115 \mathrm{~m} / \mathrm{s}
$$

\begin{tabular}{ccrrrr} 
Projectile & Balle & \multicolumn{3}{c}{ Bille } \\
- & - & & - & \\
- Température d'impact $\left({ }^{\circ} \mathrm{C}\right)$ & 975 & 20 & 850 & 1100 \\
$\begin{array}{c}\text { Durée d'oxydation à } 1100^{\circ} \mathrm{C} \\
\text { après impact (h) ......... }\end{array}$ & 24 & 120 & 72 & 192
\end{tabular}

Ces transformations progressives, dont la rapidité dépend des conditions particulières d'oxydation, influent sur le pouvoir de protection et déterminent la durée de vie.

Les deux tests cycliques $\mathrm{C}$ et $\mathrm{E}$, qui représentent deux cas d'oxydation très différents, servent d'exemple :

$\mathrm{C}$ : séjour prolongé à température élevée,

$\mathrm{E}$ : cyclage très lent avec un séjour assez long dans le domaine de températures moyennes.

4.1 Evolution de la composition chimique. 4.1.1 Oxydation de la zone extérieure du revêtement. - Examen métallographique. - Pendant la première phase de l'oxydation, la phase Sn-Al forme un film d'alumine à la surface du revêtement. Au cours des changements de température, et plus particulièrement lors des refroidissements, des fissures apparaissent dans ce film d'alumine. Elles se réparent au cours du chauffage suivant par formation nouvelle d'alumine.

Pendant les tests cycliques, on observe donc une consommation accélérée de l'alliage Sn-Al par oxydation.

Dans le test E (Fig. 18), on constate la disparition complète de la phase liquide après 30 cycles environ. A ce stade, les pores de la zone extérieure du revêtement et les fissures sont remplis d'un oxyde de composition complexe.

Par contre, dans le test $\mathrm{C}$, la zone extérieure, remplie d'oxyde, contient dans sa partie intérieure et dans ses fissures de la phase Sn-Al qui n'est pas oxydée, même après une durée prolongée d'oxydation (Fig. 19). On n'observe un épuisement total de la phase $\mathrm{Sn}-\mathrm{Al}$ qu'après un nombre de cycles dépassant environ 200.

Analyses à la microsonde. - L'examen à la microsonde des éléments présents dans les oxydes révèle :

Pour le test C :

Après 89 cycles (Fig. 20), des oxydes riches en chrome et en titane, contenant également les éléments 


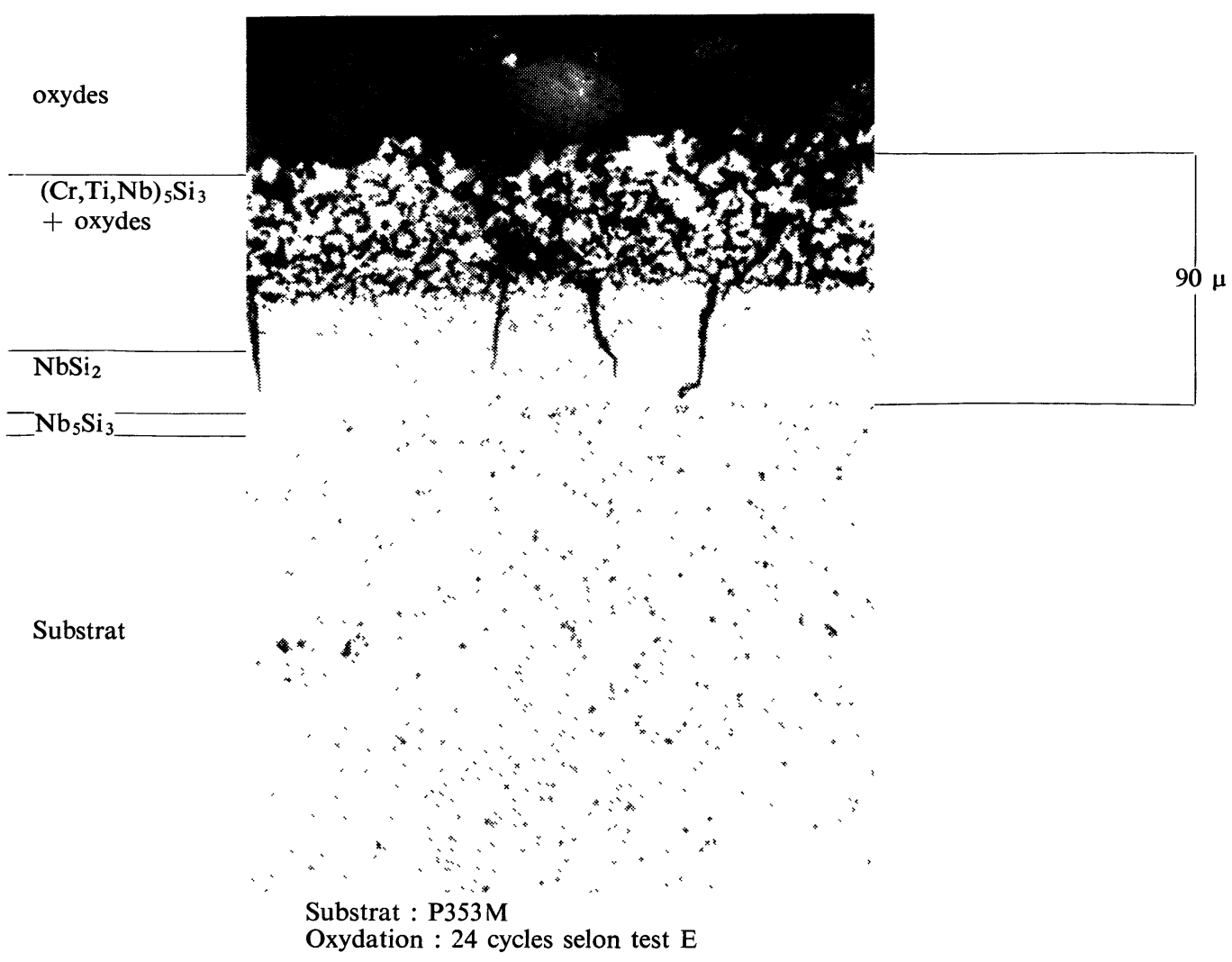

FIG. 18. - Revêtement de siliciure $\mathrm{Cr}, \mathrm{Ti}, \mathrm{Nb} / \mathrm{Sn}-\mathrm{Al}$ après oxydation cyclique.

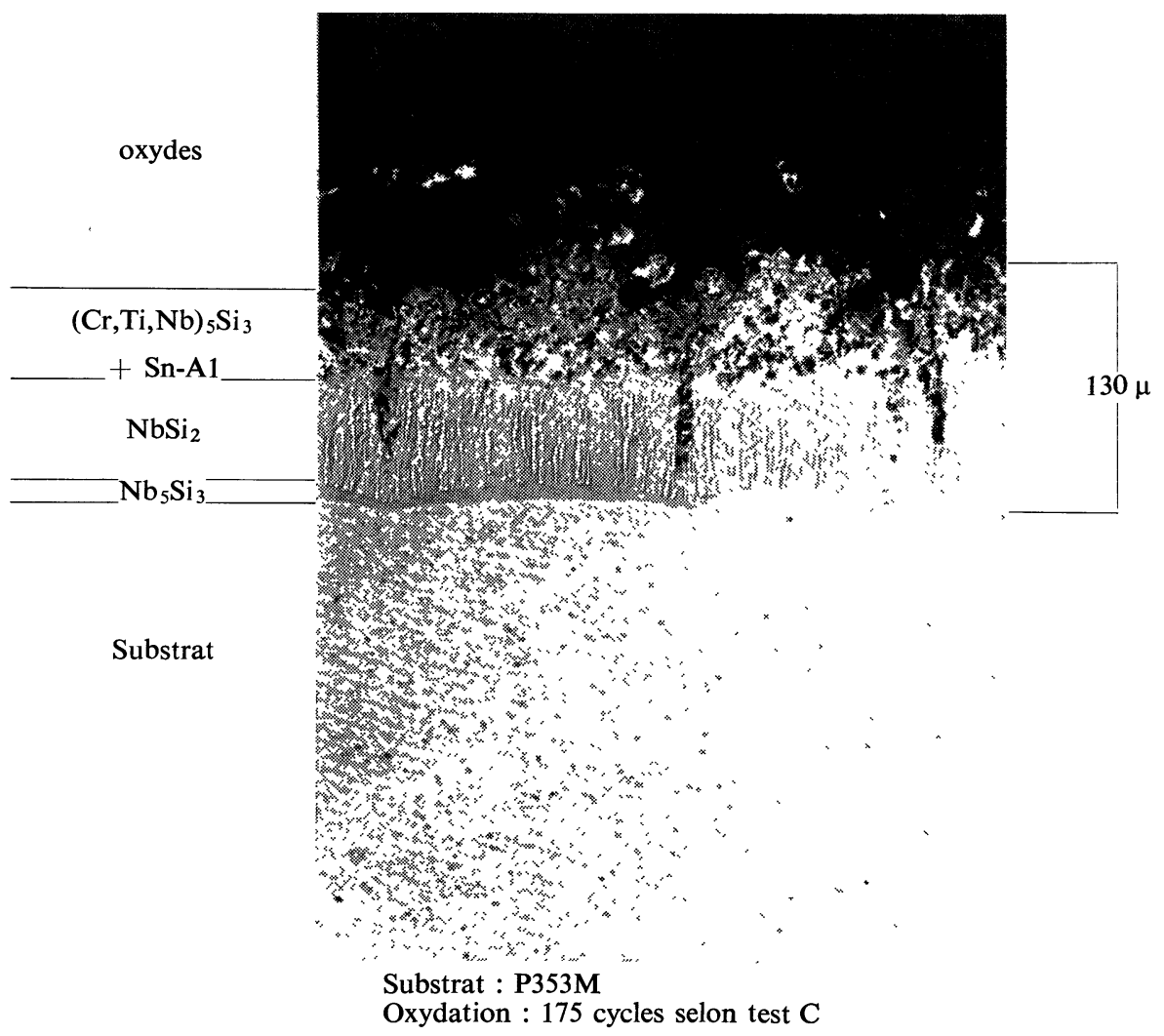

FIg. 19. - Revêtement de siliciure $\mathrm{Cr}, \mathrm{Ti}, \mathrm{Nb} / \mathrm{Sn}$ - $\mathrm{Al}$ après oxydation cyclique.

$\mathrm{Al}, \mathrm{Sn}, \mathrm{Si}$ et $\mathrm{Nb}$, couvrent la zone extérieure et poreuse du revêtement.

A l'intérieur de cette zone, on note la présence de
Sn-Al. D'après l'examen métallographique, le Sn-Al peut être présent en partie sous forme d'oxyde, en partie sous forme d'alliage non oxydé entièrement 

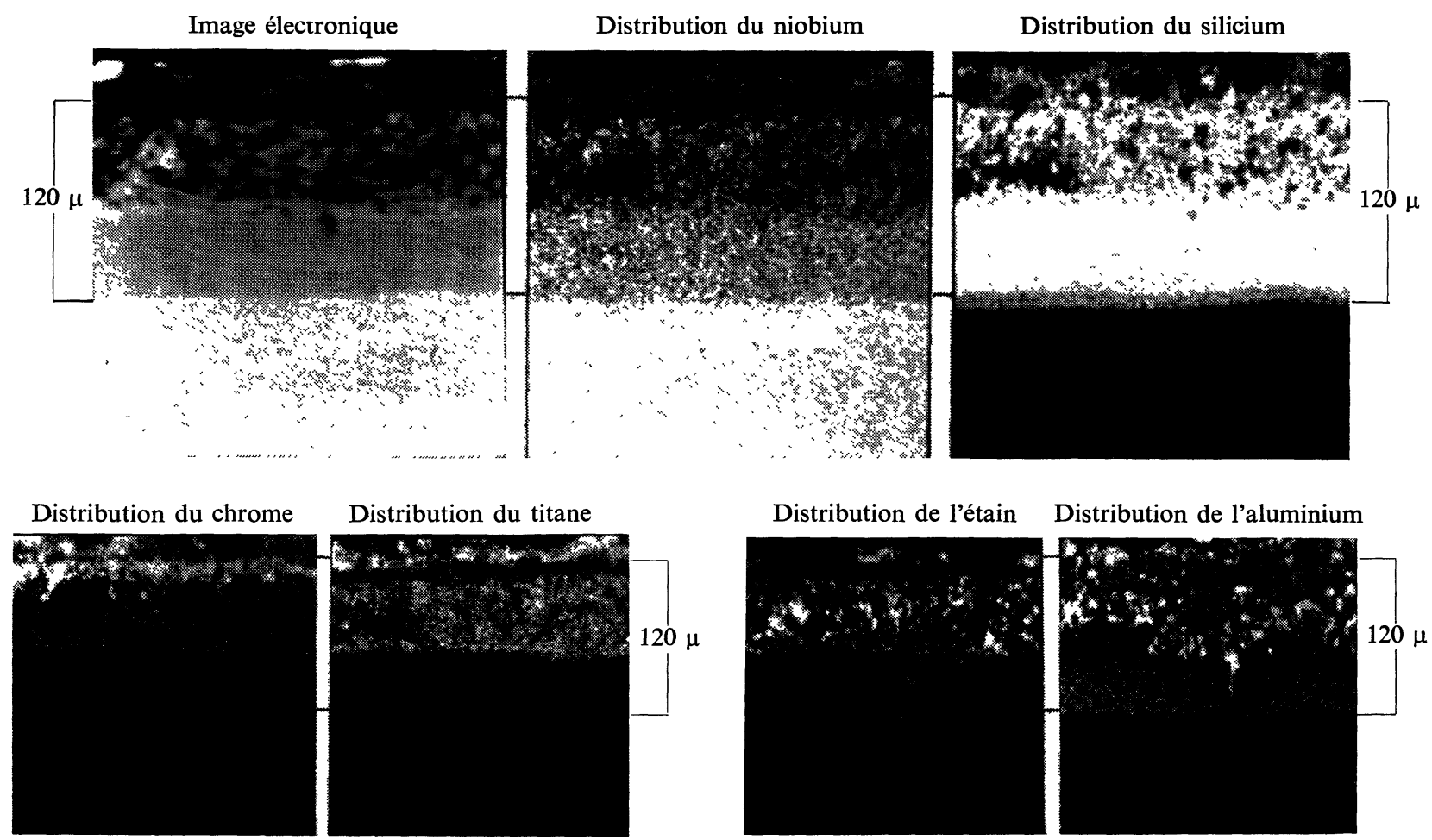

FIG. 20. - Concentration des éléments dans un revêtement de siliciure $\mathrm{Cr}, \mathrm{Ti}, \mathrm{Nb} / \mathrm{Sn}$ - $\mathrm{Al}$ après oxydation cyclique. Test C, 89 cycles.

inclus dans les pores du siliciure. Le siliciure de la zone extérieure semble être privé de chrome.

Dans les oxydes remplissant les fissures, on décèle la présence de $\mathrm{Si}, \mathrm{Sn}, \mathrm{Nb}$ et de traces de $\mathrm{Al}$.

Pour le test E :

Après 47 cycles (Fig. 21), la surface du revêtement est constituée surtout d'un film d'alumine contenant également de l'oxyde d'étain. La zone extérieure poreuse $\mathrm{du}$ siliciure est encore riche en chrometitane et contient peu d'aluminium. Les fissures sont remplies d'oxydes qui contiennent $\mathrm{Sn}, \mathrm{Si}, \mathrm{Nb}$ et parfois $\mathrm{Ti}$.

4.1.2 Zone intérieure $d u$ revêtement. - Dans les cas des tests $\mathrm{C}$ et $\mathrm{E}$, on constate qu'il n'y a pas de changement essentiel de la composition de la zone de $\mathrm{Nb}_{5} \mathrm{Si}_{3}$ et de $\mathrm{NbSi}_{2}$ après oxydation prolongée. Une diffusion du chrome ou du titane de la zone extérieure vers la zone intérieure de $\mathrm{NbSi}_{2}$ n'a pas lieu.

4.1.3 Oxydes présents dans les couches de siliciure non imprégnées. - Les figures 22 et 23 montrent des couches de siliciure de $\mathrm{Cr}, \mathrm{Ti}, \mathrm{Nb}$ et de $\mathrm{Cr}, \mathrm{Fe}, \mathrm{Nb}$ qui n'ont pas été imprégnées de $\mathrm{Sn}-\mathrm{Al}$ et qui ont subi 120 cycles du test $\mathrm{C}$.

Dans le premier cas, l'oxyde qui couvre la surface contient du titane et du silicium. Le chrome est présent dans la partie intérieure de cette couche d'oxyde. Dans le deuxième cas, l'oxyde couvrant le silicium contient du chrome, du niobium et du silicium.

Dans les deux cas, on note une consommation de la zone extérieure par oxydation et de nombreuses fissures qui sont remplies d'oxydes. Elles n'atteignent cependant pas le substrat.
Il est possible que les oxydes protecteurs formés par le siliciure, également dans le cas de couches imprégnées, contribuent essentiellement à la protection du revêtement pendant et après épuisement de Sn-Al.

4.2 Evolution de la structure. - 4.2.1 Variations d'épaisseur des zones de siliciure (Fig. 24). - Après fabrication, les trois zones superposées de siliciure possèdent les épaisseurs moyennes suivantes :

$\begin{array}{lr}\mathrm{Nb}_{5} \mathrm{Si}_{3} & 9 \mu \\ \mathrm{NbSi}_{2} & 17 \mu \\ (\mathrm{Cr}, \mathrm{Ti}, \mathrm{Nb})_{5} \mathrm{Si}_{3} & 55 \mu\end{array}$

La diffusion, lors d'une oxydation à température élevée, entraîne des changements progressifs de l'épaisseur de ces zones. Cela est particulièrement prononcé dans le test $\mathrm{C}$, dont chaque cycle comprend un traitement thermique plus intense qu'un cycle du test $\mathrm{E}$ (4 cycles du test $\mathrm{E}$ correspondent à environ 1 cycle du test $\mathrm{C}$, en ce qui concerne l'effet de diffusion).

Les zones $\mathrm{Nb}_{5} \mathrm{Si}_{3}$ et $\mathrm{NbSi}_{2}$ montrent une croissance tandis que l'épaisseur de la zone extérieure alliée diminue légèrement.

La croissance de $\mathrm{NbSi}_{2}$ peut s'expliquer par une oxydation préférentielle du chrome et du titane dans la zone extérieure.

On note, en effet, une porosité accrue de la zone extérieure (Fig. 25) après une oxydation de longue durée dans le test $\mathrm{C}$.

4.2.2 Fissuration. - Apparition des fissures. - Les revêtements soumis au test $\mathrm{C}$ montrent une faible tendance à la fissuration dans la zone intérieure 

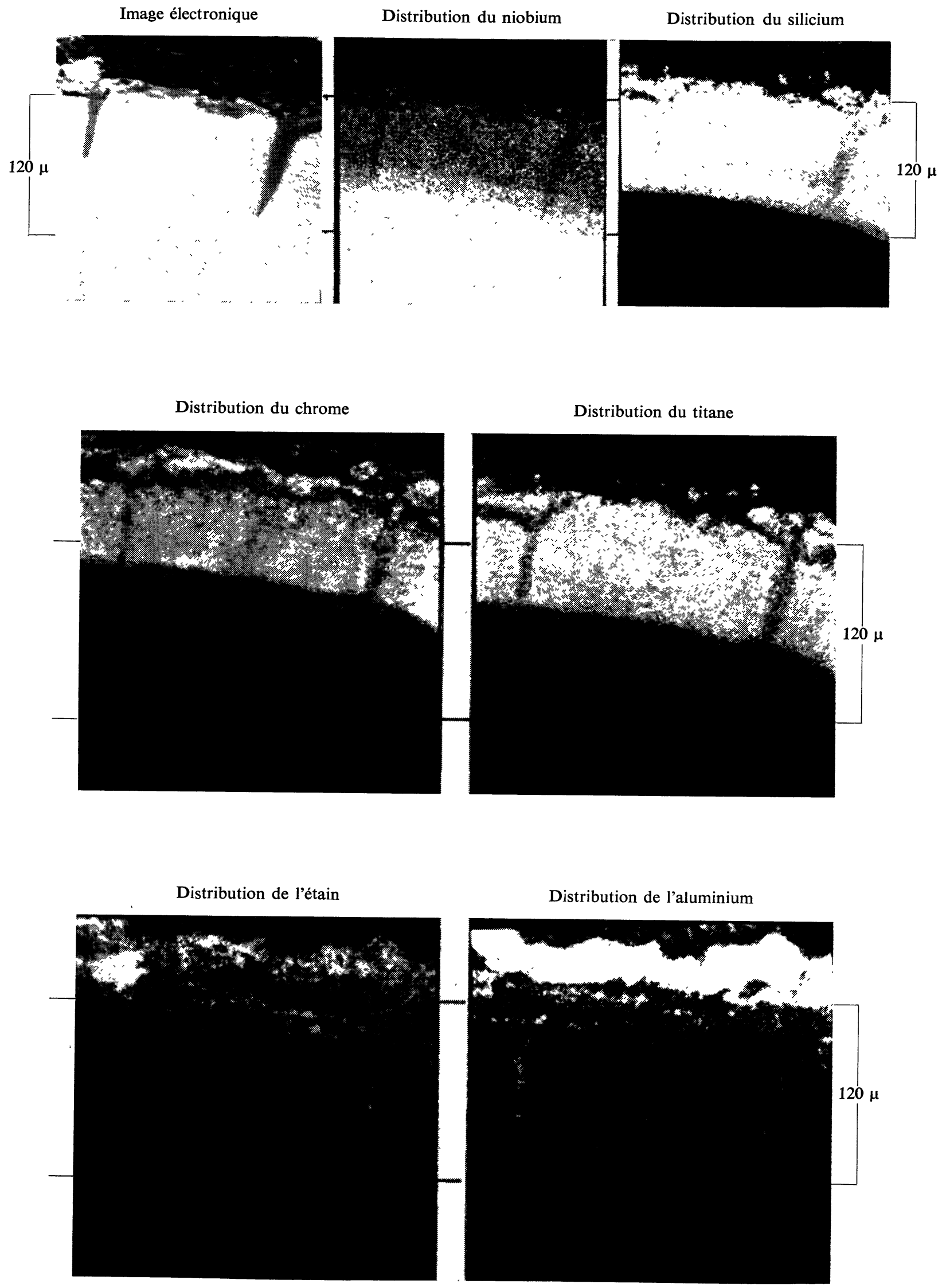

FIG. 21. - Concentration des éléments dans un revêtement de siliciure $\mathrm{Cr}, \mathrm{Ti}, \mathrm{Nb} / \mathrm{Sn}$ - $\mathrm{Al}$ après oxydation cyclique. Test E, 47 cycles. 


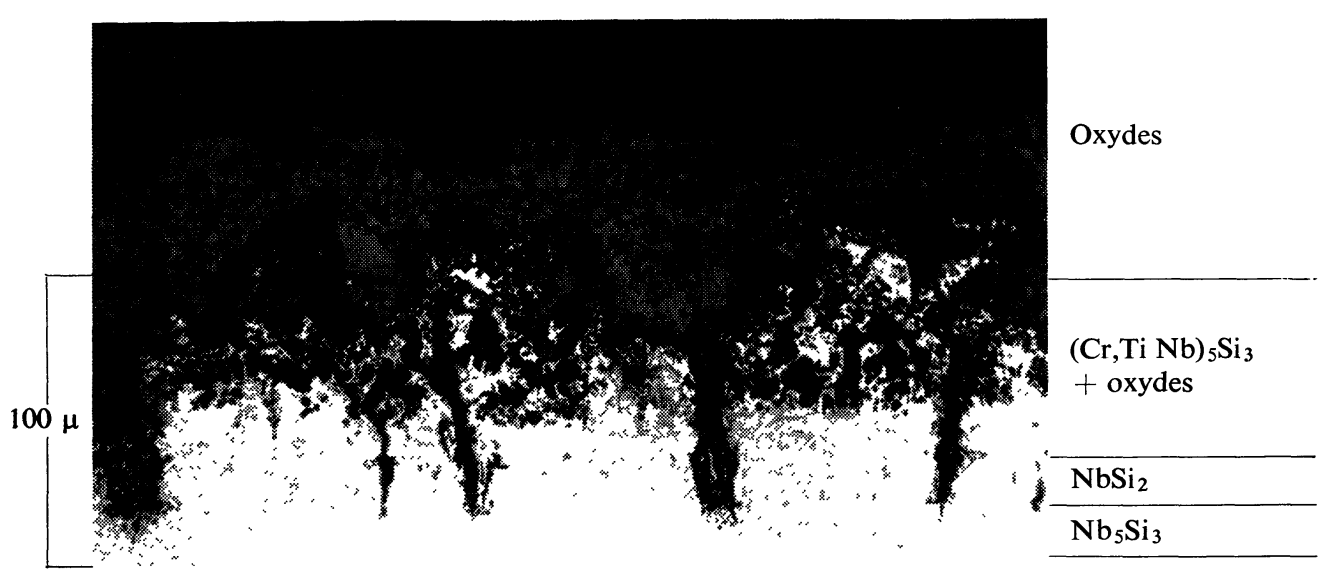

Substrat

Substrat : P353M Oxydation : 124 cycles selon test $\mathrm{C}$

Fig. 22. - Revêtement de siliciure $\mathrm{Cr}, \mathrm{Ti}, \mathrm{Nb}$ non imprégné après oxydation cyclique.

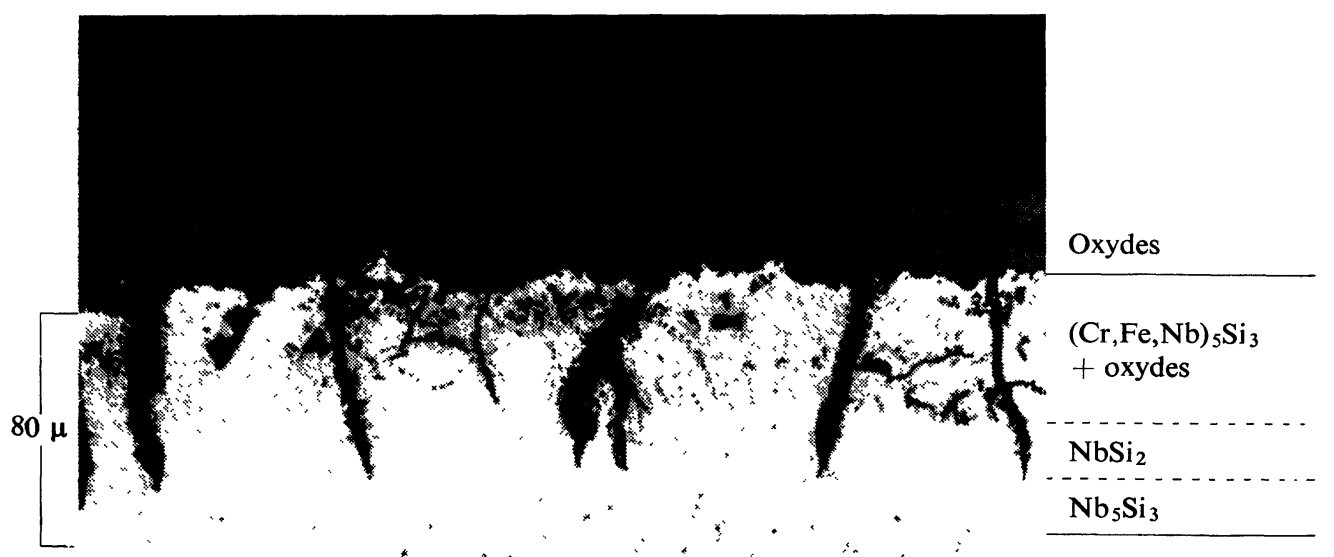

u

Substrat : P353M Oxydation : 120 cycles selon test $\mathrm{C}$

Fig. 23. - Revêtement de siliciure $\mathrm{Cr}, \mathrm{Fe}, \mathrm{Nb}$ après oxydation cyclique.

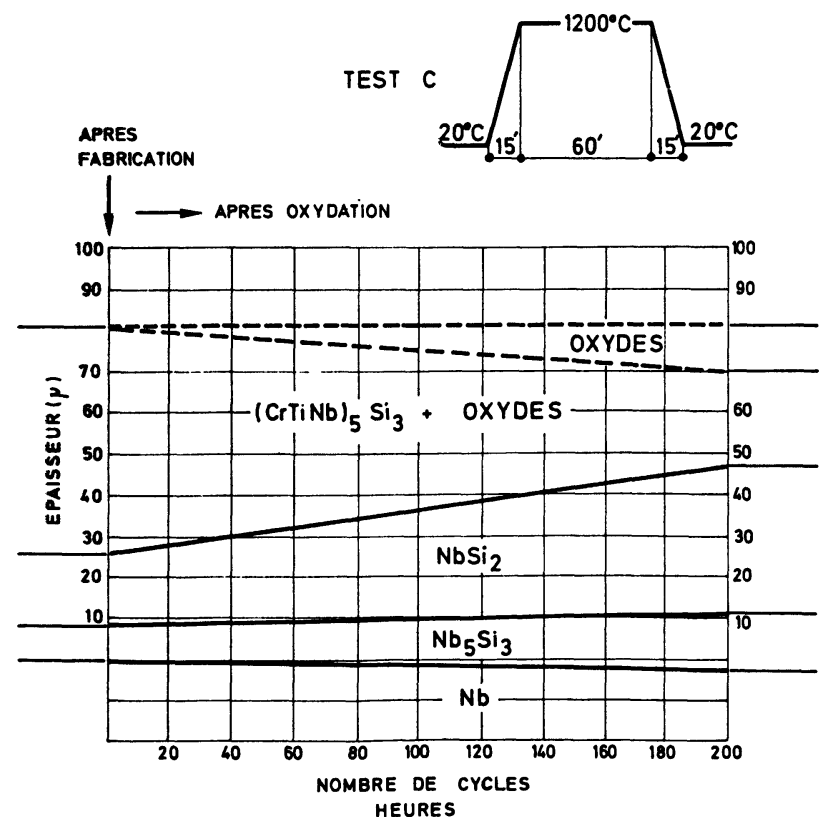

FIG. 24. - Variation de l'épaisseur des zones de siliciure pendant l'oxydation. compacte de $\mathrm{NbSi}_{2}$. La présence de $\mathrm{Sn}-\mathrm{Al}$ dans la zone extérieure conduit à la réparation des fissures (Fig. 19). Même après épuisement presque complet de $\mathrm{Sn}-\mathrm{Al}$, le nombre des fissures demeure restreint et elles sont étroites (Fig. 25).

Ce n'est pas le cas pour le test E. Après une oxydation rapide de la phase $\mathrm{Sn}-\mathrm{Al}$, la couche de siliciure présente de nombreuses fissures verticales qui se propagent souvent dans la zone intérieure de $\mathrm{Nb}_{5} \mathrm{Si}_{3}$ du revêtement et qui atteignent parfois l'interface $\mathrm{Nb}_{5} \mathrm{Si}_{3} /$ substrat.

Observations relatives d̀ la racine des fissures. Un phénomène très intéressant apparaît au niveau de la racine des fissures. La figure 26 en représente un exemple typique. La zone de $\mathrm{Nb}_{5} \mathrm{Si}_{3}$ s'épaissit sous la racine des fissures. Il semble que la vitesse de formation du $\mathrm{Nb}_{5} \mathrm{Si}_{3}$ soit supérieure à la vitesse de propagation de la fissure. Les fissures n'atteignent donc jamais le substrat. Ce cas de croissance de la zone de $\mathrm{Nb}_{5} \mathrm{Si}_{3}$ sous la racine apparaît dans les tests $\mathrm{C}, \mathrm{D}, \mathrm{E}$ sur un substrat de niobium pur, déjà avant 50 cycles. 


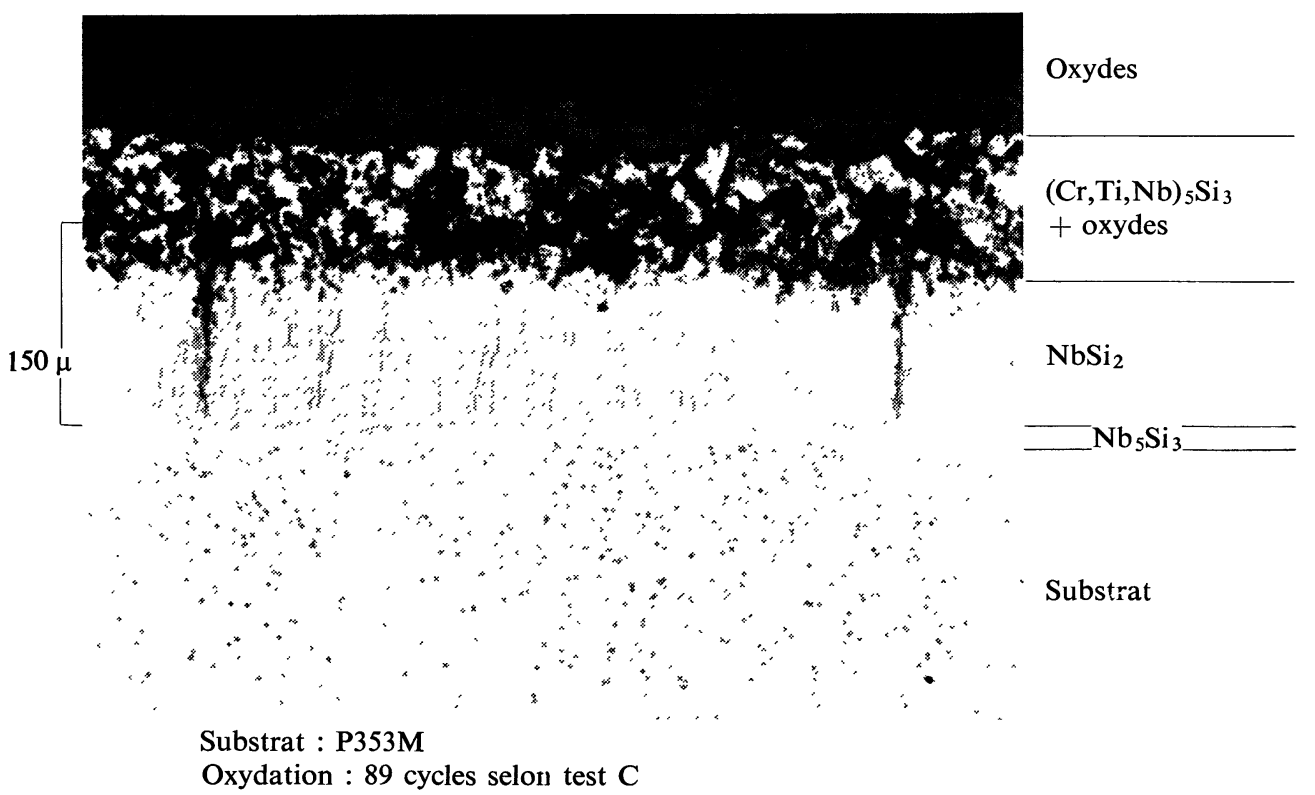

FIG. 25. - Revêtement de siliciure $\mathrm{Cr}, \mathrm{Ti}, \mathrm{Nb} / \mathrm{Sn}-\mathrm{Al}$ après oxydation cyclique.

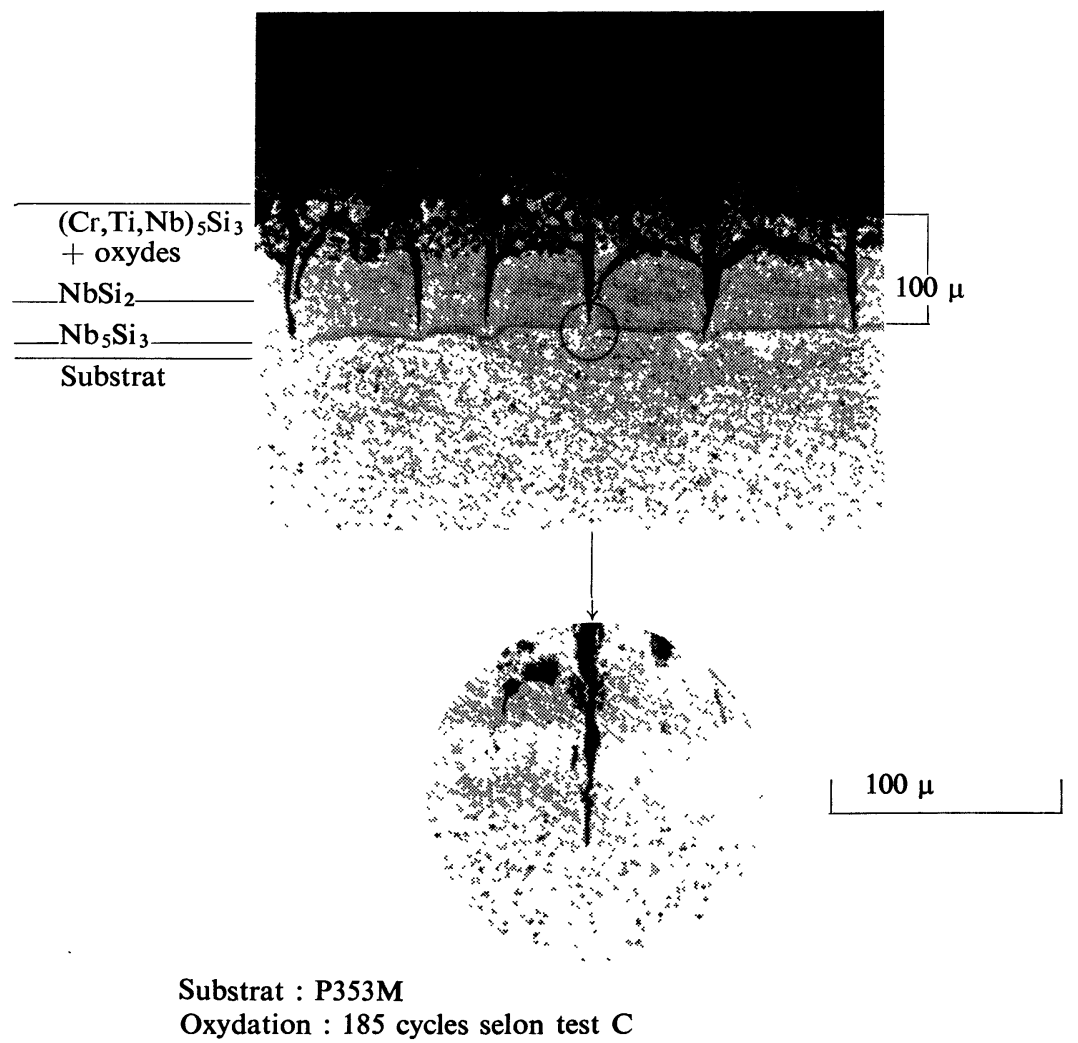

Fig. 26. - Revêtement de siliciure $\mathrm{Cr}, \mathrm{Ti}, \mathrm{Nb} / \mathrm{Sn}$ - $\mathrm{Al}$ après oxydation cyclique.

Pour les couches déposées sur un substrat de P $353 \mathrm{M}$, ce phénomène n'est visible qu'après plus de 50 cycles pour les tests $D$ et $E$.

Il semble qu'il existe au fond de la fissure une source de silicium qui permet une croissance radiale de la couche de $\mathrm{Nb}_{5} \mathrm{Si}_{3}$ dans le niobium. Or, la diffusion du silicium est plus rapide à la surface qu'à l'intérieur du siliciure. On peut donc supposer que le silicium, fourni par la zone de $\mathrm{NbSi}_{2}$, profite de l'interface créée par la fissure et présente une diffusion accélérée le long des parois de la fissure jusqu'au fond de celle-ci.
4.3 Discussion des résultats. - Dans les revêtements de siliciure de $\mathrm{Cr}, \mathrm{Ti}, \mathrm{Nb}$ imprégnés de $\mathrm{Sn}-\mathrm{Al}$, l'endurance du revêtement est fonction de plusieurs facteurs interdépendants :

1. Présence d'un film protecteur d'oxyde à la surface du revêtement.

2. Présence de $\mathrm{Sn}-\mathrm{Al}$ dans la zone extérieure qui assure l'autorépartition des fissures de la zone intérieure compacte.

3. Vitesse de pénétration de l'oxygène le long des 
fissures remplies d'oxydes et résistance à l'oxydation de la zone intérieure du revêtement.

4. Arrêt des fissures au niveau de la zone de $\mathrm{Nb}_{5} \mathrm{Si}_{3}$.

Les deux premiers facteurs jouent le rôle le plus important pendant la première phase d'oxydation et les deux derniers déterminent la durabilité du revêtement en cas d'apparition des fissures.

Pendant une oxydation cyclique qui comprend un séjour prolongé à température élevée (conditions du test $\mathrm{C}$ ), le revêtement a un pouvoir de protection accru.

Les facteurs suivants peuvent expliquer ce phénomène :

- Des oxydes de chrome-titane situés dans la zone extérieure du revêtement contribuent à la protection. Une plasticité éventuelle de ces oxydes à température élevée peut favoriser l'étanchéité de la zone extérieure à l'infiltration d'oxygène.

- La consommation de Sn-Al par oxydation serait donc ralentie. De plus, le traitement thermique provoque la fermeture d'une partie de la porosité, ce qui donne naissance à des inclusions de Sn-Al dans le siliciure qui ne sont pas exposées à l'oxydation.

- Action d'auto-réparation plus efficace à température élevée.

- Une augmentation plus rapide de l'épaisseur de toute la zone de $\mathrm{Nb}_{5} \mathrm{Si}_{3}$ qui éloigne le substrat de la racine des fissures et la croissance particulière du $\mathrm{Nb}_{5} \mathrm{Si}_{3}$ sous la racine des fissures peuvent favoriser l'arrêt des fissures à l'intérieur du revêtement.

La faible endurance du revêtement dans des conditions de cyclage lent, dans le domaine de températures moyennes (test E) s'explique par les raisons suivantes :

- Le pouvoir d'auto-réparation de la phase liquide pendant les passages à des températures moins élevées est réduit.

- L'oxydation de la phase $\mathrm{Sn}-\mathrm{Al}$ est rapide, ce qui conduit à son oxydation totale après peu de cycles.

- L'effet d'auto-réparation est alors supprimé et il se forme de nombreuses fissures profondes.

- Or, la croissance locale de la phase de $\mathrm{Nb}_{5} \mathrm{Si}_{3}$ sous les racines ne se produit pas pendant les premiers cycles car elle nécessite à température moyenne un séjour prolongé $\left(^{5}\right)$.

Conclusion. - Pour réaliser un revêtement qui résiste pendant une centaine d'heures à une oxydation à température élevée, en dépit de refroidissements

(5) Ce phénomène n'a été observé dans ce test que dans le cas exceptionnel où les revêtements ont résisté à un nombre important de cycles (Fig. 26). fréquents, un système de protection a été développé qui associe une couche de siliciure de niobium allié de $\mathrm{Cr}, \mathrm{Ti}$ à un alliage de $\mathrm{Sn}-\mathrm{Al}$ liquide à la température d'oxydation.

Les avantages de ce système apparaissent sur trois plans :

1) Diffusion. - Grâce à la couche de siliciure, la diffusion du revêtement dans le métal de base est lente.

2) Oxydation. - Le film d'alumine formé à la surface du siliciure par l'alliage $\mathrm{Sn}-\mathrm{Al}$ contribue à la formation de barrières qui s'opposent à l'infiltration de l'oxygène.

3) Auto-réparation. - L'interaction siliciure/Sn-Al liquide à l'intérieur de la couche permet, à température élevée et dans certaines conditions, la réparation des fissures qui se forment dans la zone intérieure compacte du siliciure. Cet effet de réparation s'exerce déjà pendant l'imprégnation, ce qui diminue le risque d'une défaillance du revêtement causée par des défauts locaux de fabrication.

La résistance du revêtement au cyclage entre une température élevée et la température ambiante dépend fortement des vitesses de refroidissement et de chauffage.

Dans des conditions de cyclage très lent qui comprennent des passages de plusieurs heures dans le domaine des températures moyennes, le pouvoir de protection du revêtement est très réduit. Cela s'explique par une oxydation rapide de la phase de $\mathrm{Sn}-\mathrm{Al}$ et par l'absence d'auto-réparation à basse température.

La vulnérabilité du revêtement aux basses et moyennes températures ne nuit pas à sa résistance à haute température dans la mesure où l'on en tient compte en évitant les chauffages et les refroidissements lents.

L'examen de la structure du revêtement après un nombre important de cycles comprenant des changements de température plus rapides et des séjours plus longs à température élevée révèle la présence d'oxydes de chrome et de titane dans la zone extérieure du revêtement. Ces oxydes contribuent probablement à ralentir l'oxydation du Sn-Al, ce qui permet de maintenir le pouvoir d'auto-réparation du revêtement.

Indépendamment de l'action de l'étain-aluminium, un mécanisme d'arrêt des fissures au niveau de la zone de $\mathrm{Nb}_{5} \mathrm{Si}_{3}$ semble jouer un rôle important dans la durabilité du revêtement.

Les auteurs tiennent à remercier la Société Nationale d'Etude et de Construction de Moteurs d'Aviation, qui a effectué certains tests d'oxydation cyclique, les tests d'impacts et les tests de choc thermique. Nous remercions tout particulièrement $M$. Brunetaud pour la permission de publier ces résultats. 


\section{Bibliographie}

[1] GADD (J.), Oxidation resistant coating for columbium alloys, Communication personnelle, 1968.

[2] Lux (B.), Stecher (P.), Perlhefter (N.), Protection contre l'oxydation du niobium au moyen d'une couche de $\mathrm{NbSi}_{2}$ imprégnée de $\mathrm{Sn}-\mathrm{Al}$, Annales des Mines, 1966, 802.

[3] Stecher (P.), Lux (B.), Funk (R.), Protection contre l'oxydation d'alliages de niobium, Entropie, 1968, 21, 39.

[4] Gillot (J.), Stecher (P.), Lux (B.), Stabilité mécanique d'une phase liquide comprise dans un revêtement composite de protection du niobium contre l'oxyda- tion, Publication à paraître simultanément avec le présent texte.

[5] Priceman (S.) et Sama (L.), New protective coating allow use of refractory metal alloys in reusable reentry vehicles, SAE Journal, 1967, 75, 46.

[6] Gauje (G.), Grammagnac (J.), Chevillon (J.), Rapport technique SNECMA, YOTM. 3 No. 30415 , 9.1.1969.

Résultats d'essais effectués sur l'alliage de niobium $P 353$ M protégé par le procédé Battelle avec et sans sous-couche SNECMA.

Compte rendu YOT No. 23553, 8.4.1969.

Conférence Niobium, Paris, 1969. 